\title{
Unsupervised cellular phenotypic hierarchy enables spatial intratumor heterogeneity characterization, recurrence-associated microdomains discovery, and harnesses network biology from hyperplexed in-situ fluorescence images of colorectal carcinoma
}

Samantha A. Furman ${ }^{1}$, Andrew M. Stern ${ }^{1,2, *}$, Shikhar Uttam ${ }^{1,+}$, D. Lansing Taylor ${ }^{1,2}$, Filippo Pullara $^{3}$, S. Chakra Chennubhotla ${ }^{1,2,3, *}$

${ }^{1}$ Department of Computational and Systems Biology, University of Pittsburgh, PA, 15260, USA

${ }^{2}$ University of Pittsburgh Drug Discovery Institute, University of Pittsburgh, Pittsburgh, PA, 15261, USA

${ }^{3}$ Splntellx, Inc., 2425 Sidney St, Pittsburgh, PA 15203

*Corresponding Author Emails: chakra@spintellx.com (S. Chakra Chennubhotla), sternam@pitt.edu (Andrew M. Stern) 


\section{Abstract}

LEAPH is an unsupervised machine learning algorithm for characterizing in situ phenotypic heterogeneity in tissue samples. LEAPH builds a phenotypic hierarchy of cell types, cell states and their spatial configurations. The recursive modeling steps involve determining cell types with low-ranked mixtures of factor analyzers and optimizing cell states with spatial regularization. We applied LEAPH to hyperplexed (51 biomarkers) immunofluorescence images of colorectal carcinoma primary tumors $(\mathrm{N}=213)$. $\mathrm{LEAPH}$, combined with pointwise mutual information (PMI), enables the discovery of phenotypically distinct microdomains, composed of spatially configured computational phenotypes. LEAPH identified a subset of microdomains visualized as the spatial configuration of recurrence-specific signaling networks whose intracellular and intercellular interactions support cancer stem cell maintenance and immunosuppression in the evolving tumor microenvironment. The LEAPH framework, when combined with microdomain discovery and microdomain-specific network biology, has the potential to provide insights into pathophysiological mechanisms, identify novel drug targets and inform therapeutic strategies for individual patients. 


\section{Introduction}

Tumor cells and their stromal cell counterparts that comprise the tumor microenvironment (TME) reciprocally coevolve to generate heterocellular communication networks. A distinctive characteristic of the functional organization of this continuously evolving ecosystem is spatial intratumoral heterogeneity (ITH), a key determinant of disease progression landmarks in multiple carcinomas that include colorectal cancer [1-10]. Therefore, to optimize diagnosis, prognosis, therapeutic strategies and to identify novel therapeutic targets it is important to define spatial ITH in the tumors of individual patients and determine the mechanistic underpinnings of its relationship to metastatic potential, immune evasion, recurrence, therapeutic response and drug resistance. $[1,3,11,12]$.

The first step in investigating spatial ITH is to identify the cell phenotypes within the TME. This, however, is a challenging task owing not only to the diversity of well-defined cell types within the TME but also the intrinsic plasticity of many of these cell types in response to the selection pressures within their particular confines (i.e., microdomains) [13]. Several single cell approaches are well suited to capture this emergent phenotypic continuum, but at the expense of preserving spatial context. Thus, complementary experimental and computational approaches are needed to further define spatial ITH. The choice of construction of a cellular phenotyping algorithm is based on the type of data generated due to the steep tradeoff between cellular resolution, spatial context, and the number of genomic/proteomic probes employed [1416]. There has been a recent explosion of hyperplexed [17] (> 9 fluorescence or mass specbased) biomarker labeling and imaging modalities utilizing various reagent technologies to probe the same tissue sections with several dozens of biomarkers at cellular and subcellular resolutions [18-20]. The challenge now is to accurately characterize the complex spatial and high-dimensional output of these hyperplexed techniques. 
Single-cell phenotyping analysis exists in various forms, each with advantages and drawbacks. With high-dimensional data, the analysis usually involves dimensionality reduction but observations have been made that cellular subpopulations distinct in multidimensional space overlap visually in the reduced two-dimensional space [21-24]. Common drawbacks of singlecell clustering algorithms include: 1) reliance on supervised methods leading to user-defined cellular phenotypes as opposed to data-driven cellular phenotypes, 2) lack of a spatial context due to the limitations of data generation, 3) use of hard clustering (a cell belongs to one and only one cellular phenotype) which leaves no room to identify cells along a phenotypic continuum. In Table S1, we detail the current state-of-the-art cellular phenotyping methods and analysis studies within the past decade and specify the incorporation of spatial context, soft clustering, hierarchical component, and unsupervised techniques. To our knowledge, there does not exist a cellular phenotyping method and spatial analysis framework that is unsupervised, harnesses the spatial context, and promotes the phenotypic continuum with sensitivity for identifying transitional state cell populations from hyperplexed in-situ fluorescence images.

In this paper, we propose LEAPH, an unsupervised machine learning algorithm for characterizing cellular phenotypic heterogeneity in a tumor microenvironment (Figure 1). LEAPH builds a phenotypic hierarchy of cell types, cell states and their spatial configurations. We applied LEAPH on hyperplexed (51 biomarkers) immunofluorescence images of colorectal carcinoma $(C R C)$ primary tumor tissue samples $(N=213)$. The biomarkers were selected with the interest of sampling a range of CRC and cancer biology TME properties (see Data section).

The data-driven and computationally unbiased approach of LEAPH captured a phenotypic hierarchy comprised of specialized, transitional, and multi-transitional cell states that are intrinsic to the architecturally complex and reciprocally co-evolving TME of CRC. Each LEAPH derived computational phenotype $(\mathrm{CP})$ is further characterized by a unique biomarker signature for ease of interpretation. With these biomarker signatures, we conclude properties 
demonstrating the heterogeneity of immune cells, cancer stem cells, tumor cells, and cancer associated fibroblasts. In addition, we performed a virtual simulation where the tissue is assumed to be labeled with a subset of key biomarkers (as opposed to the 51 biomarkers we started with) to demonstrate the process to obtain a rank-order subset of biomarkers for characterizing phenotypic diversity.

Using our previous work utilizing pointwise mutual information (PMI) to characterize spatial ITH [25], we show how LEAPH enables the discovery of microdomains, which are spatial configurations of computational phenotypes driving disease progression dynamics in the TME of CRC. With a microdomain-specific network biology approach using partial correlation network analysis described in our previous work [26], we find microdomains where the cancer stem cells are driving a Wnt-signaling based immunosuppressive program in patients that will experience recurrence within 3 years post-surgical resection. The LEAPH framework, when combined with microdomain discovery and spatial network biology, has the potential to provide mechanistic insights into disease biology, inform therapeutic strategies for individual patients, identify novel targets for drug discovery and support prognostic/diagnostic predictive analyses.

\section{Results}

\section{Hyperplexed fluorescence colorectal cancer (CRC) data}

The primary source of data for this study, is a cohort of 213 CRC tissue samples (TMA core size $=0.6 \mathrm{~mm}, 1$ sample per patient) hyperplexed using Cell DIVE (GE Life Sciences, Issaquah, WA) [18] (Figure 1a). Currently, there exists multiple hyperplexing technologies, each with unique properties. Hyperplex imaging techniques can be non-destructive with the methodology of antibody labeling being either iterative [18] or performed in a batch prior to imaging [19]. There exists other hyperplexing technologies unrelated to fluorescence imaging such as mass cytometry which are both destructive and perform biomarker batch labeling [20]. 
For this study, our data is imaged using Cell DIVE (GE Life Sciences, Issaquah, WA) [18] which involves non-destructive cyclical immunofluorescence labeling with two or three antibodies labeled with distinct fluorescent probes, followed by imaging and subsequent quenching of the fluorescence. This process is repeated to capture all the required antibodies (biomarkers). The data consists of image stacks taken at each region of interest and overall image stack consists of several images for each of several imaging rounds. Each round includes a nuclear (DAPI) image that is used as a reference for registering all the images from all the rounds. Quantitation of images in each round includes the fluorescence intensity of each measured biomarker. Images are also acquired after quenching rounds for the purpose of autofluorescence removal [18]. Validation of this process shows robustness and preservation of biomarker stability and biological integrity [18, 27-31]. Processing of Cell DIVE images includes correction for uneven illumination across the field of view, removal of autofluorescence, registration, and automated quality control (QC) detection of several categories of defects, including failed registration, blurred or saturated images, and other imaging issues.

The images and data undergo a series of tissue and cell quality checks, log2 transformation and normalization steps. To integrate data from batch processing each biomarker is normalized to a control median. Cellular segmentation is done using a collection of structural biomarkers: NaKATPase (cell membrane, cell border), S6 (cytoplasm), and DAPI (nucleus) (Figure S1). Cells are filtered using individual QC scores generated for each cell (scores less than 0.7-0.8 will not be included indicating inaccurate registration, misalignment, or tissue loss) and based on number of pixels per segmented subcellular compartment. See Methods for more information on data generation and pre-processing steps.

The biomarkers chosen are protein markers for specific cell lineages, oncogenes, tumor suppressors, and post-translational protein modifications indicative of cellular activation states [18] (Figure S2, Table S2). A subset of the biomarkers was selected specifically based on their 
known association with CRC. In addition to the tissue samples, the CRC patient data set also includes clinical information for each patient regarding sex, age, chemotherapy treatment, and days until recurrence post-surgical resection (patient statistics in Table S3).

\section{LEAPH builds a phenotypic hierarchy of cell types and states through recursive steps of probabilistic clustering and spatial regularization}

LEAPH can process a range of input data from a single sample ( $2 \mathrm{~K}$ cells) to a cohort of samples (tested on 213 tissue samples, $\sim 500 \mathrm{~K}$ cells total). LEAPH applies recursive steps of modeling cell types with low-ranked mixtures of factor analyzers (MFA) and cell states with spatial regularization to account for the spatial context around each cell (Figure 1b). The ownership probabilities for any given cell are constructed recursively by parsing through the hierarchy. Our stopping criterion for recursion is to apply thresholds on the angle between the subspaces created by the mixture models and the fraction of cells with a majority ownership probability in each cluster to ensure that the hyperplexed dataset is not being overfitted (see Methods). The terminal nodes of the tree, i.e., leaves, signify distinct data-driven CPs discovered in the input dataset and form the components of the final MFA model determined by the recursive decomposition (Figure 1c).

We define cell states as being specialized or non-specialized based on the CP ownership probabilities for each cell. A specialized cell state is defined by a strong propensity towards a single CP (ownership probability $>0.95$ ). For convenience, we further group the non-specialized cell state as being a transitional (ownership probabilities spread between two CPs) or multitransitional (ownership probabilities spread across more than two CPs). We can visualize the spatial distribution of the resulting CP's within a tissue sample by assigning each cell to the CP with the highest ownership probability (Figure 1c). 
To illustrate the process of discovering phenotypic hierarchy, we generated synthetic biomarker data (Figure 2a) to reflect the distribution of biomarker values in the hyperplexed CRC data. In particular, there is a varying spread of biomarker values likely due to the individual biomarker response sensitivity (Figure S4) and the observation of dominant phenotypes (e.g., epithelial and stromal cells) with various nested cell subtypes (e.g., immune, cancer stem cells). To automate the process of phenotypic discovery, we use a recursive probabilistic approach with a low-dimensional latent space mixture of factor analyzers (MFA) (See Methods for more details). Each mixture component is a factor analyzer in a two-dimensional latent space, as we observed this is sufficient to capture the input variance (Figure S5). On the synthetic data (Figure 2a topleft), we instantiate a one-tier MFA model with four mixture components (Figure 2a top-right) and our proposed recursive decomposition where each level of the hierarchy identifies two dominant mixture components (Figure 2a bottom). Comparing the results to the ground truth synthetic data, the one-tier MFA model is unsuccessful at discovering the four phenotypes. The recursive decomposition method separates the larger broad clusters in the first level and the finer sub-clusters at the second level of the hierarchy.

The MFA model parameters are learned through random initializations over a hundred different runs and a consensus set of model parameters are inferred to capture the optimal subspace representation (Figure S6). The recursive probabilistic clustering when applied along results in a large and unrealistic population of non-specialized cells (typically $25 \%$ in a single tissue sample). The soft clustering is agnostic to the spatial complexity of the TME, a key component driving ITH. Based on properties of spatial ITH and the spatial tissue architecture of a tumor, we expect neighborhoods of cells to be spatially coherent (e.g., epithelial/tumor cells to be surrounded by, or spatially proximal to, other epithelial/tumor cells, but making allowance for the presence of tumor-infiltrating lymphocytes and other stromal cells) [32]. To filter false-positive 
non-specialized cells, we add a spatial regularization component to the recursive probabilistic clustering.

The novel objective function for the spatial regularization component consists of two terms: one to promoting ownership probability confidence and one to promote spatial coherence within a small neighborhood. We assume the two terms hold equal weight and calculate the tuning parameter accordingly. All cells are tested under this objective function but only the cells classified as non-specialized initially (ownership probability 0.05-0.95) are further optimized. Cells initially classified as specialized are fixed (remain invariant during the optimization) to avoid altering the ownerships of cells such as tumor-infiltrating lymphocytes which will not maintain spatial coherence. On a single tissue sample, we track the ownership probabilities of the cells undergoing spatial regularization. We observe that most of the cells converge to one cluster, but a subset of cells remains non-specialized (1-4\% in a single tissue sample) (Figure 3b). To gain a deeper spatial understanding, we demonstrate the transformation of ownership probabilities of two cells in this simulation. One cell conforms to the surrounding homogenous neighborhood and one cell remains non-specialized between the two clusters due to the heterogeneous nature of the surrounding neighborhood (Figure 3c). A more detailed description of LEAPH can be found in the Methods section.

\section{LEAPH captures a diverse set of computational phenotypes having distinct biomarker signatures comprised of specialized, transitional, and multi-transitional cell states and enables the visualization of their spatial configurations}

We applied LEAPH on the CRC hyperplexed data ( $~ 500 \mathrm{~K}$ cells) and identified a cellular phenotypic heterogeneity tree consisting of 13 distinct CP's (leaves) (Figure 3a). We characterize each CP with a unique biomarker signature to describe the intrinsic biological properties (Figure 3b). For each CP, the biomarkers are classified as either "ON", "OFF”, or "Non-Specific" ("NS”) (see Methods, Figure S7). With the biomarker signatures, we identify a 
heterogeneous population of epithelial (CP1-7) and stromal (CP8-13) cell types. We can further classify some phenotypes as tumor cells (CP1-3), cancer stem cells (CP5), cancer associated fibroblasts (CP9, CP12), macrophages (CP8), and immune cells (CP10, 13) based on specific indicative biomarkers (Table S2). A small subset of non-specialized cells (7\% of $\sim 500 \mathrm{~K})$ are dispersed across the 13 CP's identified.

To visualize the spatial complexity, we assign cells a CP identify based on the highest ownership probability and systematically select representative patients from each outcomestage based cohort (e.g., NED-Stage I, NED-Stage II, ... etc - see Methods for selection process) (Figure 3c). As expected, each tissue sample is comprised of a heterogeneous population of CP's comprised of specialized, transitional, and multi-transitional cell states (Figures 1c, 3d). In addition to applying LEAPH to the entire patient cohort, we can apply LEAPH to sub-cohorts of patients (particularly based on diagnosis: Stage I, II, III). We do not find a relationship between each stage-specific set of CP's and recurrence-outcome, but we did notice a difference visually in the spatial distributions. In the next section, we further pursue the spatial relationships between the 13 LEAPH-derived CP's within each tissue sample to time-torecurrence and find that the spatial distributions of CP-pairs are statistically significant in relation to time-to-recurrence.

\section{LEAPH derived computational phenotypes enable the discovery of recurrence- associated microdomains with pointwise mutual information and determine the microdomain-specific network biology}

Visually, each tissue sample is composed of a unique pattern of computational phenotypes (from LEAPH). We hypothesize that the patient cohorts with the greatest differences in outcomes will also have distinct spatial heterogeneity patterns. To discover these spatial patterns, we pooled two different cohorts: 1) patients who exhibit recurrence under 3 years (REC-3yrs, N=46) and 2) patients who exhibit no-evidence-of-disease beyond 8 years (NED- 
8yrs, N=45) (Table S3, see Methods). We use pointwise mutual information (PMI) to quantify the spatial co-occurrence of each CP-pair within each tissue sample. The resulting PMI values are either negative, zero, or positive indicating if a CP-pair spatially co-occurs below, the same, or above average compared to a random background distribution (Figure 4a, see Methods for details).

For each CP-pair, we aggregate the PMI values to form NED-8yrs and REC-3yrs distributions (Figure S9). We use the Wilcoxon rank sum test and Kendall-rank correlations (and p-values) to determine which CP-pairs have a significant difference in spatial co-occurrence between the NED-8yrs and REC-3yrs patients (Table S4, Methods). We report the Kendall-rank correlation between the PMI values and time-to-recurrence in Figure $4 \mathrm{~b}$ as well as the $p$-value and results of the Wilcoxon rank sum test in Table S4. In addition, we perform the same statistical analysis using the clinical variables (stage, grade, sex, age) and CP fractions per tissue sample. We found that the spatial distributions (PMI covariates) are statistically more significant in relation to time-to-recurrence than the CP fractions per tissue sample and are similar to the correlation between stage/grade with time-to-recurrence.

We report 5 CP-pairs which spatially co-occur statistically more significantly in the REC-3yrs patient cohort than the NED-8yrs cohort (Figure 4b). The 5 CP-pairs consist of 6 CP's and form two microdomains (Figure 4c). Microdomain $1\left(\mathcal{M}_{1}\right)$ consists of a self-paired CP with heterogeneous properties suggesting the presence of both tumor cells (PCK26+) and tumor associated macrophages (TAMs) (CD163+, Figure S8). The presence of CD163 suggests a protumorigenic M2 polarization [33]. Microdomain $2\left(\mathcal{M}_{2}\right)$ is comprised of an epithelial-stromal network with cancer associated fibroblasts (CAFs) (CP9) as the "hub" interconnecting with an immune cell phenotype (CP13), cancer stem cell phenotype (CP5), and two heterogenous epithelial phenotypes (CP6, CP7) (Figure S8). The association of the stromal cells, particularly CAFs, with poor survival in CRC has been consistently demonstrated [34-36]. 
We further utilize partial correlation network analysis to investigate the microdomain-specific differential network biology driving the spatial contingency to recurrence outcome (see Methods). The partial correlation measures the true correlation between two biomarkers after the linear dependence from other biomarkers is removed [37, 38]. We perform a connectivity analysis using a permutation test to determine the most significant and differential $\left(99^{\text {th }}\right.$ percentile) biomarker pairwise partial correlations between the NED-8yrs and REC-3yrs cohorts (see Methods, Table S5 and S6) [38]. In Figure 4d, we visualize the outcome-specific differential biomarker pairwise networks, where each edge color and width correspond to the corresponding partial correlation value. Additionally, the biomarkers are color-coordinated based on the properties and functions in Table S2 and Figure S2.

In $\mathcal{M}_{1}$, the differential biomarker pairwise partial correlations (Table S5) confirm the presence of cancer stem cells and TAMs supported by Wnt signaling and PI3K/AKT/MTOR in a hypoxic environment as suggested by crosstalk of the later with carbonic anhydrase 9 (CA9). Importantly, each of the partial correlations among the biomarker pairs in $\mathcal{M}_{1}$ are significantly different when a comparison is made between the NED-8yrs versus the REC-3yrs cohorts. In fact, a majority of these comparisons show a change in sign of the partial correlations suggesting that a distinct difference in network dysregulation in addition to co-occurrence of cell type per se is necessary for driving the recurrence phenotype. The network we have observed for $\mathcal{M}_{1}$ (Figure $4 \mathrm{~d}$, Table S5) is consistent with recurrence and the crucial role Wnt signaling plays in cancer stem cell maintenance and tumor cell induced-immunosuppression in CRC and other cancer types [39-47].

Likewise for $\mathcal{M}_{2}$, in addition to the aforementioned tumor cell-stromal cell spatial configuration with CAFs as a critical hub (Figure 4c), the difference between the partial correlations among the biomarker pairs in the NED-8yrs versus the REC-3yrs cohorts (Table S6) indicate the critical evolution of network signaling required for driving the recurrence phenotype (Figure 4d). 


\section{Optimal selection of biomarkers for reproducing phenotypic diversity}

For hyperplexing platforms that are non-destructive of the tissue and iterative in labeling biomarkers, such as the Cell DIVE platform, there is an option of bringing in biomarkers on demand. In other words, instead of using 51 biomarkers upfront, can we select an optimal list of biomarkers much smaller in number that we can apply iteratively to reveal the phenotypic diversity?

To test this hypothesis, we performed a virtual simulation of systematically introducing biomarkers into the data and applying LEAPH at the first (epithelial-stromal dissection) and second (epithelial- and stromal- subtyping dissection) levels of the hierarchy (see Methods). For comparison, we use the maximum ownership probability from the ALL-DATA results with 51 biomarkers (Figure 3) as the ground truth CP identity. We measure the accuracy as the percentage of cells with a matching $\mathrm{CP}$ identity to the ground truth.

At the first level, only 2 biomarkers (pck26, E-cadherin) are needed to reproduce the cellular phenotypic assignments with an accuracy above 97\% (Figure S10). Increasing the number of biomarkers further increases the accuracy to almost 99\% (Figure S10). This analysis demonstrates that the epithelial-stromal dissection is a low-dimensional dissection, as suspected.

At the second level of the hierarchy, both the epithelial and stromal subtyping dissections require a larger set of biomarkers than level one (Figure S10). The epithelial subtyping dissection reaches $89 \%$ accuracy with 4 biomarkers (4EBP1, pS6, pMAPKAPK2, PCNA) (Figure S10). An increase in biomarkers does not make an overall substantial difference in the reproduction accuracy. The stromal subtyping dissection reaches above $93 \%$ accuracy with 4 biomarkers (Lamin A/C, Claudin 1, Akt, 4EBP1). Contrasting from the epithelial subtyping 
dissection, the addition of more biomarkers leads to a convergence to almost $97 \%$ accuracy with 8 or more biomarkers (Figure S10).

We conclude that there exists an optimal subset of biomarkers at each LEAPH dissection. This virtual simulation demonstrates the capabilities of LEAPH to exploit the non-destructive and iterative nature of the Cell DIVE platform with a parsimonious selection of biomarkers to regenerate identical computational phenotypes (Figure 5).

\section{Discussion}

We have proposed LEAPH, an unsupervised, spatially informed, probabilistic recursive clustering method, to exploit the spatial ITH properties prominent in many cancer types and other diseases. The unsupervised and spatially informed approach using biomarker data enables the tumor architecture to drive the discovery of computational phenotypes. Additionally, rather than limiting cells to identify with a single cell type, we exploit the cellular phenotypic continuum with probabilistic clustering and spatial regularization to further classify cell states as specialized or non-specialized. Biologically, the non-specialized cells (transitional and multitransitional) represent cells undergoing a transformation (e.g., epithelial-mesenchymaltransition, cell-fusion) between CPs. LEAPH does not computationally identify the direction of the transition.

LEAPH is built as a generalizable model amenable to other data sources, soft clustering algorithms, and spatial regularization objective functions/optimization methods. The input criteria for LEAPH is single cell data vectors with corresponding spatial information per sample. Other sources for spatial multiparameter cellular and sub-cellular imaging data include transmitted light (H\&E and IHC), fluorescence, immunofluorescence, live cell biomarkers, mass spectrometry, spatial transcriptomics, electron microscopy etc. The soft clustering step of LEAPH is amenable to other probabilistic mixture models such as but not limited to gaussian 
mixture models [48] and mixtures of probabilistic principal component analysis [49]. An argument could be made that a low-dimensional model will be scalable with a larger number of biomarkers. In addition to the choice of a low-dimensional model, there is room for improvements to better estimate the noise model based on the data generation method. For the spatial regularization step, the objective function in place is amenable to other optimization methods (e.g., gradient descent) and the objective function is adjustable to other sources of input data.

Beyond LEAPH, we have also laid out a framework utilizing the LEAPH derived CPs to investigate the systems biology of TMEs in relation to disease progression. We use PMI to compute the pairwise relative likelihood of spatial co-occurrence between CPs and subsequently form microdomains based on the recurrence-specific CP pairs. We hypothesize that capturing the higher order spatial relationships between groups of phenotypes may provide additional information on the spatial configurations of the microdomains. Further, there are many graphical models [50-52] that could also be used in the microdomain-specific network biology analyses.

In this study, we applied LEAPH on hyperplexed immunofluorescence images of CRC primary tumor tissue samples and identify 13 data-driven CP's comprised of specialized, transitional, and multi-transitional cell states. We characterized each $\mathrm{CP}$ with a biomarker signature and concluded properties demonstrating the heterogeneity of epithelial and stromal cell types. We further used PMI to discover two microdomains with spatial distributions significantly different between patients in the NED-8yrs and REC-3yrs cohorts based on the Kendall rank correlations and Wilcoxon rank sum test.

The two microdomains identified here, $\mathcal{M}_{1}$ and $\mathcal{M}_{2}$ are both more significantly associated with the REC-3yrs cohort than the NED-8yrs cohort. These microdomains comprise spatial configurations of tumor/stromal CPs consistent with those inferred from bulk transcriptomic 
analyses and resultant consensus molecular signatures that are associated with poor outcome $[35,53]$. Nevertheless, these microdomains are also seen in the NED-8yrs cohort suggesting that if the spatial configurations of the CPs within these microdomains are directly involved in recurrence, other factors are also necessary. Importantly, the partial correlation analysis of biomarker pairs showed a strikingly significant difference between the two patient cohorts for both $\mathcal{M}_{1}$ and $\mathcal{M}_{2}$. This result supports our working hypothesis that within the evolving tumor microenvironment, the molecular signaling networks within both $\mathcal{M}_{1}$ and $\mathcal{M}_{2}$ undergo a regulatory switch to confer a recurrence phenotype supported by cancer stem cell maintenance and immunosuppression [39, 40, 42, 44-47, 54-59]. Thus, $\mathcal{M}_{1}$ and $\mathcal{M}_{2}$ microdomains represent the spatial manifestation of emergent recurrence-specific networks. To further investigate our findings, we can exploit the non-destructive property of Cell DIVE ${ }^{\text {TM }}$-like imaging platforms that allow iterative analysis of additional biomarkers on the same tissue sample to test mechanistic hypotheses, identify novel biomarkers and optimal therapeutic strategies (see below and Figure 5).

Here, we denoted the leaves of the tree generated by LEAPH as our CPs. However, information at any level of the LEAPH hierarchy can be used for the spatial analysis described in this paper. In fact, in our previous work [26], we have used only the first level of the LEAPH hierarchy (epithelial, stromal and epi-stroma interface domains) to successfully predict the risk of 5-year recurrence in $\mathrm{CRC}$. Indeed, the same analysis can be repeated by using the entire library of CPs derived from LEAPH. We expect the predictive analysis will be further enhanced with the additional phenotypes and the derived microdomains. Based on the statistical robustness of our approach and external experimental validation of our results found in literature, we predict that we will find similar microdomains presented here when our methods are applied on larger CRC datasets. 
The selection of biomarkers is a key factor in determining the resulting phenotypes that will emerge. We demonstrated the existence of an optimal set of biomarkers capable of reproducing the CPs derived from LEAPH with the entire biomarker set. Our virtual simulation provides evidence for performing iterative cycles of imaging and computational analysis with an optimal biomarker set to fully exploit the capabilities of LEAPH in combination with a non-destructive multi to hyperplexed imaging platform.

LEAPH bridges an important knowledge gap in the analytical frameworks that we previously proposed $[25,26]$. In particular, LEAPH builds a statistical framework for the application of PMI [25] in the unsupervised discovery of data-driven microdomains. With this advancement, we can fully exploit the spatial network biology based analysis from our previous work [26] to provide mechanistic insights into disease biology such as the generation of outcome-specific new pathways, new cellular phenotypes, and iterative experimental testing of additional biomarkers of the same microdomains (Figure 5). We can generate hypothesis for future experimental and computational studies to further investigate the signaling and cross talk of this immunosuppressive program across the TME landscape to understand the biology of CRC recurrence and possible therapeutic strategies. Systematically deciphering the microdomainspecific network biology will allow us to not only a priori predict recurrence and its aggressiveness resulting in personalized patient surveillance but also potentially select optimal therapeutic interventions by identifying dominant risk-associated microdomains (Figure 5). This framework forms the basis of an explainable Al platform [60] with applications probing and modulating tumor environment including prognostics, diagnostics, patient stratification for clinical trials, drug target identification, patient-specific therapeutic strategies including immunotherapy, as well as animal toxicology studies (Figure 5). 


\section{Acknowledgements}

This work was supported in part by the grants NIH/NCI U01CA204836 (SCC, DLT); NIH/NIBIB 5T32EB009403 and 1NIH/NCI 1F31CA254332 (SF); NIH P30CA047904, and PA DHS 4100054875 (DLT); NIH/NHGRI U54HG008540, and UPMC Center for Commercial Applications of Healthcare Data 711077 (SCC). The authors are very grateful to Dr. Fiona Ginty and her team members at GE Global Research, including Dr. Christopher J. Sevinsky, Dr. Yousef Al-Kofahi and Brion Sarachan, for their collaborative support and for sharing the hyperplexed colorectal cancer dataset generated on Cell DIVE ${ }^{\mathrm{TM}}$ from their previous study [18].

\section{Competing Interests:}

S.C. C. and D.L.T. have ownership interest in Splntellx, Inc., a computational and systems pathology company. S.C.C is on a leave of absence from the University of Pittsburgh. The other authors disclose no potential conflicts of interest. The computational and systems pathology intellectual property is owned by the University of Pittsburgh and is exclusively licensed to Splntellx Inc., Pittsburgh, PA.

\section{Author contributions}

Conceived and designed the study - S.A.F., A.M.S., D.L.T., F.P., S.C.C.

Wrote the paper - S.A.F., A.M.S., D.L.T., F.P., S.C.C.

Developed computational platform - S.A.F., F.P., S.C.C.

Performed formal analysis - S.A.F., F.P., S.C.C.

Developed systems biology framework - S.A.F., A.M.S., F.P., S.C.C.

Data quality control and providing analysis support - S.A.F., S.U., F.P., S.C.C.

Technical issues and edited and reviewed the paper - S.A.F., A.M.S., S.U., D.L.T., F.P., S.C.C. 


\section{References}

1. Almendro, V., A. Marusyk, and K. Polyak, Cellular heterogeneity and molecular evolution in cancer. Annu Rev Pathol, 2013. 8: p. 277-302.

2. Bussard, K.M., et al., Tumor-associated stromal cells as key contributors to the tumor microenvironment. Breast Cancer Res, 2016. 18(1): p. 84.

3. Caswell, D.R. and C. Swanton, The role of tumour heterogeneity and clonal cooperativity in metastasis, immune evasion and clinical outcome. BMC Med, 2017. 15(1): p. 133.

4. DeGregori, J., Connecting Cancer to Its Causes Requires Incorporation of Effects on Tissue Microenvironments. Cancer Res, 2017. 77(22): p. 6065-6068.

5. Hanahan, D. and R.A. Weinberg, Hallmarks of cancer: the next generation. Cell, 2011. 144(5): p. 646-74.

6. Roerink, S.F., et al., Intra-tumour diversification in colorectal cancer at the single-cell level. Nature, 2018. 556(7702): p. 457-462.

7. Shia, J., et al., Morphological characterization of colorectal cancers in The Cancer Genome Atlas reveals distinct morphology-molecular associations: clinical and biological implications. Mod Pathol, 2017. 30(4): p. 599-609.

8. Tape, C.J., The Heterocellular Emergence of Colorectal Cancer. Trends Cancer, 2017. 3(2): p. 79-88.

9. Tauriello, D.V.F. and E. Batlle, Targeting the Microenvironment in Advanced Colorectal Cancer. Trends Cancer, 2016. 2(9): p. 495-504.

10. Tauriello, D.V., et al., Determinants of metastatic competency in colorectal cancer. Mol Oncol, 2017. 11(1): p. 97-119.

11. Balkwill, F.R., M. Capasso, and T. Hagemann, The tumor microenvironment at a glance. 2012, The Company of Biologists Ltd. 
12. Junttila, M.R. and F.J. de Sauvage, Influence of tumour micro-environment heterogeneity on therapeutic response. Nature, 2013. 501(7467): p. 346-354.

13. Smith, E.A. and H.C. Hodges, The Spatial and Genomic Hierarchy of Tumor Ecosystems Revealed by Single-Cell Technologies. Trends in cancer, 2019.

14. Chen, H., et al., Single-cell trajectories reconstruction, exploration and mapping of omics data with STREAM. Nat Commun, 2019. 10(1): p. 1903.

15. Efremova, M. and S. Teichmann, Computational methods for single-cell omics across modalities. Nature methods, 2020. 17(1): p. 14.

16. Lundberg, E. and G.H.H. Borner, Spatial proteomics: a powerful discovery tool for cell biology. Nat Rev Mol Cell Biol, 2019. 20(5): p. 285-302.

17. Gough, A., et al., High content analysis with cellular and tissue systems biology: a bridge between cancer cell biology and tissue-based diagnostics. The molecular basis of cancer, 2014. 4.

18. Gerdes, M.J., et al., Highly multiplexed single-cell analysis of formalin-fixed, paraffinembedded cancer tissue. Proc Natl Acad Sci U S A, 2013. 110(29): p. 11982-7.

19. Goltsev, Y., et al., Deep Profiling of Mouse Splenic Architecture with CODEX Multiplexed Imaging. Cell, 2018. 174(4): p. 968-981 e15.

20. Giesen, C., et al., Highly multiplexed imaging of tumor tissues with subcellular resolution by mass cytometry. Nature methods, 2014. 11(4): p. 417-422.

21. Amir, E.-a.D., et al., viSNE enables visualization of high dimensional single-cell data and reveals phenotypic heterogeneity of leukemia. Nature biotechnology, 2013. 31(6): p. $545-552$.

22. DiGiuseppe, J.A., et al., PhenoGraph and viSNE facilitate the identification of abnormal T-cell populations in routine clinical flow cytometric data. Cytometry Part B: Clinical Cytometry, 2018. 94(5): p. 744-757. 
23. Chen, H., et al., Cytofkit: A Bioconductor Package for an Integrated Mass Cytometry Data Analysis Pipeline. PLoS computational biology, 2016. 12(9): p. e1005112.

24. van der Maaten, L.J.P. and G.E. Hinton, Visualizing High-Dimensional Data Using tSNE. Journal of Machine Learning Research, 2008. 9(nov): p. 2579-2605.

25. Spagnolo, D.M., et al., Pointwise mutual information quantifies intratumor heterogeneity in tissue sections labeled with multiple fluorescent biomarkers. J Pathol Inform, 2016. 7: p. 47.

26. Uttam, S., et al., Spatial domain analysis predicts risk of colorectal cancer recurrence and infers associated tumor microenvironment networks. Nature communications, 2020. 11(1): p. 1-14.

27. Gerdes, M.J., et al., Emerging understanding of multiscale tumor heterogeneity. Frontiers in oncology, 2014. 4: p. 366.

28. Graf, J.F. and M.I. Zavodszky, Characterizing the heterogeneity of tumor tissues from spatially resolved molecular measures. PLoS One, 2017. 12(11): p. e0188878.

29. McKinley, E.T., et al., Optimized multiplex immunofluorescence single-cell analysis reveals tuft cell heterogeneity. JCl insight, 2017. 2(11).

30. Nelson, D.A., et al., Quantitative single cell analysis of cell population dynamics during submandibular salivary gland development and differentiation. Biology open, 2013. 2(5): p. $439-447$.

31. Santamaria-Pang, A., et al. Robust single cell quantification of immune cell subtypes in histological samples. in 2017 IEEE EMBS International Conference on Biomedical \& Health Informatics (BHI). IEEE.

32. Chen, Z., et al., Modeling Multiplexed Images with Spatial-LDA Reveals Novel Tissue Microenvironments. Journal of Computational Biology, 2020.

33. Pinto, M.L., et al., The two faces of tumor-associated macrophages and their clinical significance in colorectal cancer. Frontiers in immunology, 2019. 10: p. 1875. 
34. Calon, A., et al., Stromal gene expression defines poor-prognosis subtypes in colorectal cancer. Nature genetics, 2015. 47(4): p. 320-329.

35. Guinney, J., et al., The consensus molecular subtypes of colorectal cancer. Nature medicine, 2015. 21(11): p. 1350.

36. Isella, C., et al., Stromal contribution to the colorectal cancer transcriptome. Nature genetics, 2015. 47(4): p. 312-319.

37. Epskamp, S. and E.I. Fried, A tutorial on regularized partial correlation networks. Psychological methods, 2018. 23(4): p. 617.

38. Grimes, T., S.S. Potter, and S. Datta, Integrating gene regulatory pathways into differential network analysis of gene expression data. Scientific reports, 2019. 9(1): p. 112.

39. Augustin, I., et al., Immune cell recruitment in teratomas is impaired by increased Wnt secretion. Stem cell research, 2016. 17(3): p. 607-615.

40. Bienz, M. and H. Clevers, Linking colorectal cancer to Wnt signaling. Cell, 2000. 103(2): p. 311-320.

41. Fu, C., et al., $\beta$-Catenin in dendritic cells exerts opposite functions in cross-priming and maintenance of $C D 8+T$ cells through regulation of IL-10. Proceedings of the National Academy of Sciences, 2015. 112(9): p. 2823-2828.

42. Galluzzi, L., et al., WNT signaling in cancer immunosurveillance. Trends in cell biology, 2019. 29(1): p. 44-65.

43. Gattinoni, L., et al., Wnt signaling arrests effector $T$ cell differentiation and generates CD8+ memory stem cells. Nature medicine, 2009. 15(7): p. 808-813.

44. Grasso, C.S., et al., Genetic mechanisms of immune evasion in colorectal cancer. Cancer discovery, 2018. 8(6): p. 730-749.

45. Pai, S.G., et al., Wnt/beta-catenin pathway: modulating anticancer immune response. Journal of hematology \& oncology, 2017. 10(1): p. 101. 
46. Spranger, S. and T.F. Gajewski, $A$ new paradigm for tumor immune escape: $\beta$-catenindriven immune exclusion. Journal for immunotherapy of cancer, 2015. 3(1): p. 1-3.

47. Spranger, S. and T.F. Gajewski, Impact of oncogenic pathways on evasion of antitumour immune responses. Nature Reviews Cancer, 2018. 18(3): p. 139-147.

48. Reynolds, D.A., Gaussian Mixture Models. Encyclopedia of biometrics, 2009. 741.

49. Tipping, M.E. and C.M. Bishop, Mixtures of probabilistic principal component analyzers. Neural computation, 1999. 11(2): p. 443-482.

50. Barber, R.F. and M. Drton, High-dimensional Ising model selection with Bayesian information criteria. Electronic Journal of Statistics, 2015. 9(1): p. 567-607.

51. Foygel, R. and M. Drton. Extended Bayesian information criteria for Gaussian graphical models. in Advances in neural information processing systems. 2010.

52. Meinshausen, N. and P. Bühlmann, High-dimensional graphs and variable selection with the lasso. The annals of statistics, 2006. 34(3): p. 1436-1462.

53. Dienstmann, R., et al., Consensus molecular subtypes and the evolution of precision medicine in colorectal cancer. Nature Reviews Cancer, 2017. 17(2): p. 79.

54. De Jaeghere, E.A., H.G. Denys, and O. De Wever, Fibroblasts Fuel Immune Escape in the Tumor Microenvironment. Trends in cancer, 2019.

55. Kraman, M., et al., Suppression of antitumor immunity by stromal cells expressing fibroblast activation protein- $\alpha$. Science, 2010. 330(6005): p. 827-830.

56. Naito, Y., et al., CD8+ $T$ cells infiltrated within cancer cell nests as a prognostic factor in human colorectal cancer. Cancer research, 1998. 58(16): p. 3491-3494.

57. Turley, S.J., V. Cremasco, and J.L. Astarita, Immunological hallmarks of stromal cells in the tumour microenvironment. Nature reviews immunology, 2015. 15(11): p. 669-682.

58. Wong, P.F., et al., Multiplex quantitative analysis of cancer-associated fibroblasts and immunotherapy outcome in metastatic melanoma. Journal for immunotherapy of cancer, 2019. 7(1): p. 194. 
59. Yaeger, R., et al., Clinical sequencing defines the genomic landscape of metastatic colorectal cancer. Cancer cell, 2018. 33(1): p. 125-136. e3.

60. Tosun, A.B., et al., Explainable Al (xAl) for Anatomic Pathology. Advances in Anatomic Pathology, 2020. 27(4): p. 241-250.

61. https://www.gelifesciences.com/en/us/shop/cell-imaging-and-analysis/high-contentanalysis-systems/instruments/cell-dive-multiplexed-imaging-p-11361.

62. Ghahramani, Z. and G.E. Hinton, The EM algorithm for mixtures of factor analyzers. 1996, Technical Report CRG-TR-96-1, University of Toronto.

63. Francis, K. and B.O. Palsson, Effective intercellular communication distances are determined by the relative time constants for cyto/chemokine secretion and diffusion. Proceedings of the National Academy of Sciences, 1997. 94(23): p. 12258-12262.

64. Hallac, D., J. Leskovec, and S. Boyd. Network lasso: Clustering and optimization in large graphs. in Proceedings of the 21th ACM SIGKDD international conference on knowledge discovery and data mining. 2015. ACM.

65. Leinster, T. and C.A. Cobbold, Measuring diversity: the importance of species similarity. Ecology, 2012. 93(3): p. 477-489.

66. Wasserman, L., All of nonparametric statistics. 2006: Springer Science \& Business Media.

67. Efron, B. and T. Hastie, Computer age statistical inference. Vol. 5. 2016: Cambridge University Press.

68. Zeng, X., Y. Xia, and H. Tong, Jackknife approach to the estimation of mutual information. Proc Natl Acad Sci U S A, 2018. 115(40): p. 9956-9961.

69. Vickovic, S., et al., High-definition spatial transcriptomics for in situ tissue profiling. Nature methods, 2019. 16(10): p. 987-990. 


\section{Methods}

\section{Hyperplexed data}

The data for this study consists of 747 colorectal carcinoma (CRC) tissue samples hyperplexed using cell DIVE with 56 biomarkers measured in protein expressions plus DAPI nuclear counterstain. Cell Dive (GE Life Sciences, Issaquah, WA) [18] involves non-destructive cyclical immunofluorescence labeling with two or three antibodies labeled with distinct fluorescent probes, imaging and subsequent quenching of the fluorescence. This process is repeated to capture all the required antibodies (biomarkers). The data consists of image stacks taken at each region of interest and overall image stack consists of several images for each of several imaging rounds. Each round includes a nuclear (DAPI) image that is used as a reference for registering all the images from all the rounds. Quantitation of images in each round includes the fluorescence intensity of each measured biomarker. Images are also acquired after quenching rounds for the purpose of autofluorescence removal $[18,61]$. Processing of Cell DIVE images includes correction for uneven illumination across the field of view, removal of autofluorescence, registration, and automated quality control (QC) detection of several categories of defects, including failed registration, blurred or saturated images, and other imaging issues. The images and data undergo a series of tissue and cell quality checks, log2 transformation and normalization steps. To integrate data from batch processing each biomarker is normalized to a control median. Validation of this process shows robustness and preservation of biomarker stability and biological integrity [18, 27-31]. Images are acquired in TIFF format, while image metadata is captured in files having a simple structure that captures the provenance of which images were derived from which slides and characteristics of the acquisition [18, 61]. The biomarkers chosen are protein markers for specific cell lineages, oncogenes, tumor suppressors, and post-translational protein modifications indicative of cellular activation states (Table S2, Figure S2). The data also includes corresponding clinical information including the 
histological tumor grade, cancer stage, gender, age, and follow up monitoring for 10 years (Table S3).

\section{Data pre-processing}

\section{$\underline{\text { Cell quantitation }}$}

Cellular segmentation is done using a collection of structural biomarkers: NaKATPase (cell membrane, border), S6 (cytoplasm), and DAPI (nucleus) (Figure S1). Protein expression and standard deviation were quantified by the median biomarker intensity value within each cell mask and transformed to the log2 scale [18]. Cells are filtered using individual QC scores generated for each cell (scores less than 0.7-0.8 will not be included indicating inaccurate registration, misalignment, or tissue loss) and based on number of pixels per segmented subcellular compartment.

\section{Patient selection}

For this study, based on the clinical data, we limit the patient data set to deceased patients with recurrence within 5 years (postsurgical resection) and alive patients with no evidence of recurrence within 5 years (post-surgical resection). Further, we eliminate tissue samples with less than a threshold of 1000 cells to limit the potential adverse effects of hyperplex imaging (i.e. damaged, folded, or lost tissue). This cell threshold is computed based on the 20th percentile of number of cells per tissue sample shown in Figure S3. The final data set used is composed of 213 TMA spots (Table S3). The alternative strategies could have been to filter out cells within the damaged areas of the TMA's by examining the nuclear-to-cytosolic ratio of structural biomarkers. We chose to use the cell threshold method to preserve the unsupervised nature of LEAPH.

Biomarker selection and distributions 
We removed biomarkers showing batch affects resulting in a selection of 51 biomarkers for this analysis. The distribution of each biomarker (log2 scale) across all cells in the patient cohort is shown in Figure S4. The Kurtosis values measures the skewness of the distribution. Comparing the kurtosis values of the biomarker distributions to the kurtosis of a univariate normal distribution (kurtosis $=3$ ), many but not all these distributions can be considered to have a Gaussian shape.

\section{LEAPH: unsupervised learning algorithm for cellular phenotypic heterogeneity}

We will describe the hyperplexed dataset in a high-dimensional space, where each cell $\vec{x}(p \times 1)$ is described by a $p$ dimensional vector of biomarker expressions quantitated appropriately. Further, we assume that the hyperplexed dataset has an intrinsic low-dimensional representation. We will use a mixture of factor analyzers described by low-dimensional factor loadings $(\Lambda(p \times k))$, latent variables $(\vec{z}(k \times 1))$, mean vector $(\vec{\mu}(p \times 1))$, and noise term $(\vec{v}(p \times 1)): \vec{x}=\Lambda \vec{z}+\vec{\mu}+\vec{v}$, where $p$ is the number of biomarkers and $k$ is the low-dimension latent space [62]. The latent factors, $\vec{z}$, are generated from zero-mean, unit-variance Normal distribution $N(0, \mathrm{I})$, and the noise term, $\vec{v}$ is sampled from $N(0, \Psi)$. I is the unit variance and $\Psi$ is assumed to be a diagonal matrix. With this construction, $\vec{x}$ is distributed with zero mean and covariance $\Lambda \Lambda^{T}+\Psi[62]$.

\section{Soft clustering}

Typically, cellular phenotyping methods are constructed under the assumption that each cell belongs to one and only one cluster (hard clustering) leaving no room to identify specific cells that may belong to more than one phenotype due to an existing phenotypic continuum. With a Mixture of Factor Analyzers (MFA), we model the cells as $M$ components (clusters) with the parameters $\left(\left\{\pi_{j}, \vec{\mu}_{j}, \Lambda_{j}\right\}_{j=1}^{M}, \Psi\right)$ where $\pi_{j}$ is the component weight: $p(\vec{x})=$ $\sum_{j=1}^{M} N\left(\vec{x} \mid \vec{\mu}_{j}, \Lambda_{j} \Lambda_{j}^{T}+\Psi\right)$. We chose a two-dimensional latent space for each component in the 
MFA model, as we observed this is enough to capture the input variance (Figure S5). The expectation-minimization (EM) algorithm is utilized to estimate the model parameters [62]. The EM algorithm is initialized with a random set of parameters and the EM algorithm is not guaranteed to converge to a globally optimal solution. To account for this and ensure stability, we perform a hundred different EM optimizations, each initialized randomly. Each optimization yields an MFA model with a set of model parameters. We compute the biomarker ranking for each set of model parameters (see discriminative biomarkers section) and aggregate all biomarker rankings to compute their mean ranking. The model with a biomarker ranking closest (Euclidean distance) to the mean ranking is selected as the consensus model and deemed to provide an optimal subspace representation (Figure S6). The MFA model results in soft clustering probabilities (ownership probabilities) - each cell, $x_{c}$, holds a unique probability of belonging to each cluster $j$, denoted as $\Omega_{c j}$.

\section{Spatial regularization}

The soft clustering is agnostic to the spatial complexity of the TME, a key component driving ITH. Based on properties of spatial ITH and the spatial tissue architecture of a tumor, we expect neighborhoods of cells to be spatially coherent (e.g., epithelial/tumor cells to be surrounded by, or spatially proximal to, other epithelial/tumor cells, but making allowance for the presence of tumor-infiltrating lymphocytes and other stromal cells for example). To promote specialization in cells, we add a spatial regularization component to optimize the ownership probabilities of nonspecialized cells. The spatial regularization step optimizes the objective function which consists of two terms: ownership confidence and spatial coherence given by $: \min _{\Omega}\left(-\sum_{i=1}^{N} \sum_{j=1}^{M} \Omega_{c j} \log _{2}\left(\Omega_{c j}\right)+\lambda \sum_{(j, k)} w_{j k}|| \Omega_{j}-\Omega_{k}||_{2}\right)$. The first term minimizes the entropy of the ownership probabilities promoting specialization in cells. The second term promotes spatial coherence where $w_{j k}$ is the weight between cell $i$ and cell $j$ and is computed as the 
reciprocal of the distance between two cells: $w_{j k}=\frac{1}{\operatorname{dist}\left(\operatorname{cell}_{i}, \text { cell }_{j}\right)}$. We use a distance threshold (100 pixels at $0.5 \mu \mathrm{m} / \mathrm{pixel}$ ) to eliminate an influence between cells that are too far apart to communicate [63].

The objective function is optimized using Alternating Directions Method of Multipliers (ADMM) [64]. We assume that the probability ownership confidence (term 1) and spatial coherence (term 2) should hold equal weight and therefore compute the tuning parameter, $\lambda$, to scale term 2 to the range of term $1: \lambda=\frac{N_{o p t} \times \text { maxEntropy }}{\sum_{\{j, k\}} w_{j k}}$ where $N_{o p t}$ is the number of cells being optimized and maxEntropy is the maximum value of the entropy function (=1). Relaxing the assumption that spatial coherence and ownership confidence should hold equal weight in the objective function would lead to a larger parameter space. A higher weight for spatial coherence results in homogenous neighborhoods and a larger set of non-specialized cells. On the contrary, a larger weight for ownership confidence results in the abolishment of all non-specialized cells. We have found stable and consistent results when the tuning parameter represents an equal weighting. Cells can only have neighbors within the same tissue sample and therefore to increase computational speed and efficiency, spatial regularization is performed on each tissue sample independently.

\section{Recursive decomposition}

To automate the process of phenotypic discovery, we propose a recursive probabilistic approach where each step dissects the most dominant clusters with $M=2$ components. At each recursive step, the soft clustering step utilizes a low-dimensional latent space MFA. Subsequently, spatial regularization optimizes the resulting per-cell ownership probabilities to filter false-positive non-specialized cells by promoting ownership confidence and spatial coherence. The resulting parameters (ownership probabilities, $\Omega_{j}$, mean vector, $\vec{\mu}_{j}$, factor loadings, $\Lambda_{j}$ ) for each cluster, $j$, are passed to the next recursive step to decompose each 
cluster into further sub-clusters. This process is continued until an attempted cluster split invalidates any one of the following stopping criteria: 1) a resulting cluster takes ownership of $<$ $1 \%$ of cells, or 2 ) the angle between the mean vectors and factor loading space are both below a given threshold.

\section{Discriminative biomarker order}

Each LEAPH split results in two clusters with high dimensional mean vectors $\left(\vec{\mu}_{1}, \vec{\mu}_{2}\right)$. To determine the discriminative ordering of the biomarkers, we compute and sort the proportional difference for each biomarker $j: \Delta_{j}=\left|\vec{\mu}_{1 j}-\vec{\mu}_{2 j}\right| * \max \left(\vec{\mu}_{1 j}, \vec{\mu}_{2 j}\right)$. The absolute difference of the mean vectors may bias the selection of biomarkers with high biomarker value ranges and therefore, we opt for a proportional difference to place the biomarkers on an even level for comparison.

\section{Interpreting Computational Phenotypes}

We characterize each $\mathrm{CP}$ with a unique biomarker signature for ease of interpretation. For each biomarker and CP pair two histograms are computed: 1) histogram of biomarker values for all cells $\left(h_{A}\right)$ and 2 ) histogram of biomarker values for all specialized cells in the CP $\left(h_{B}\right)$. We compare the $20^{\text {th }}$ and $80^{\text {th }}$ percentiles of each histogram to determine the CP-biomarker classification. Each classification is as follows:

\begin{tabular}{|l|l|l|}
\hline Classification & Operator & Interpretation \\
\hline "ON" & $h_{B}^{80 t h}>h_{A}^{80 t h}$ AND $h_{B}^{20 t h}>h_{A}^{20 t h}$ & $h_{B}$ is skewed to the right \\
& & compared to $h_{A}$ \\
\hline "OFF" & $h_{B}^{20 t h}<h_{A}^{20 t h}$ AND $h_{B}^{80 t h}<h_{A}^{80 t h}$ & $h_{B}$ is skewed to the left \\
& & compared to $h_{A}$ \\
\hline
\end{tabular}




\begin{tabular}{|l|l|l|}
\hline "NS" (Non- & $h_{B}^{80 t h}>h_{A}^{80 t h}$ AND $h_{B}^{20 t h}<h_{A}^{20 t h}$ & $h_{B}$ spans across $h_{A}$ or is \\
specific) & OR & confined within $h_{A}$ \\
& $h_{B}^{80 t h}<h_{A}^{80 t h}$ AND $h_{B}^{20 t h}>h_{A}^{20 t h}$ & \\
\hline
\end{tabular}

Figure S7 depicts each distribution and $20^{\text {th }} / 80^{\text {th }}$ percentiles for each CP-biomarker pair to demonstrate how the biomarkers are classified in Figure 3b.

Selecting representative patients for visualizing computational phenotypes

Post LEAPH, we assign cells a CP identity based on the highest ownership probability value.

For $C$ CP's, we compute the fraction of each phenotype found in each patient, $i$, forming a vector $\vec{f}_{l}(C \times 1)$. When computing this fraction, we only consider the specialized cells (ownership probability $>0.95$ ) to avoid the transitional and rare cells biasing our results. For each outcome-stage-based group (e.g., NED-Stage I, NED-Stage II, NED-Stage III), we compute the average phenotype fraction vector, $\vec{f}_{a v g}$, and choose the patient closest to the mean to display in Figure 3c (Euclidean distance).

\section{Biomarker selection virtual simulation}

For comparison, we assume the LEAPH derived CP's is the ground truth. Based on the rankordered biomarkers from each LEAPH recursion step, we re-run LEAPH with the first 2, 4, .., 20 biomarkers at the first and second level of the hierarchy (Table S7). Assigning each cell to one CP based on the highest ownership probability (cell-label), we compute the accuracy of the cell-labels derived from each sub-set of biomarkers to the assumed ground truth (Figure S10). This virtual simulation demonstrates the reproducibility and capabilities to integrate LEAPH into an integrated iterative imaging and computational platform. 


\section{Discover recurrence-associated microdomains with pointwise mutual information and determine microdomain-specific network biology}

Step 1: Select patient sub-cohorts with recurrence under 3 years and with no evidence of recurrence in 8 years

To study the differences between patients at each extremum and balance the NED/REC cohorts, we prune the patient cohort further based on the time to recurrence. We consider a group of patients with no evidence of disease over 8 years (NED-8yrs, N=45) and a group of patients that exhibit recurrence under 3 years (REC-3yrs, $N=46)$. See Table $S 3$ for the patient statistics.

\section{Step 2: Construct a spatial network of interacting cells in each patient sample}

In each tissue sample, we build a spatial network where each node is a cell and the edges connect cells, say $m$ and $n$, to each other with a weight $w_{m n}=1$ if their spatial distance $d_{m n}$ is within a threshold (100 pixels/50 $\mu \mathrm{m}$, which is the same threshold used in LEAPH spatial regularization), or $w_{m n}=0$ otherwise. To remove possible artifacts, we prune each network to include only cells within the largest connected component.

Step 3: Use Pointwise Mutual Information (PMI) to quantify spatial co-occurrence between CPpairs

Pairwise association statistics, specifically pointwise mutual information (PMI), identifies microdomains consisting of one, two, or groups of phenotypes which spatially co-occur more frequently than a given background distribution. PMI maps can characterize associations at the cohort, patient, and tissue sample level and can describe both the local and global spatial heterogeneity scenarios that cannot be captured by well-known methods such as quadratic entropy [65]. 
Each cell, $c$, holds an ownership probability vector over the $M$ CP's, $\vec{\Omega}_{c}(M \times 1)$, such that $\sum_{j=1}^{M} \vec{\Omega}_{c j}=1$. The pointwise mutual information between two phenotypes, $\left(f_{i}, f_{j}\right)$, for a given network or network set, $s$, is defined as:

$$
\operatorname{PMI}_{s}\left(f_{i}, f_{j}\right)=\log _{2}\left(\frac{p\left(f_{i}^{s}, f_{j}^{s}\right)}{p\left(f_{i}^{t}\right) p\left(f_{j}^{t}\right)}\right)
$$

where $p\left(f_{i}^{s}\right)$ is the probability of phenotype $i$ occurring in a network set $s$ and $p\left(f_{i}^{t}\right)$ is the background probability distribution of phenotype $i$ (this can be an ensemble of networks or individual networks, see below). For a single patient, the joint probability is computed as:

$$
p\left(f_{i}^{s}, f_{j}^{s}\right)=\frac{1}{Z}\left(\sum_{(m, n) \in \Phi} w_{m n}\left(\vec{\Omega}_{m f_{i}} \vec{\Omega}_{m f_{j}}+\vec{\Omega}_{m f_{j}} \vec{\Omega}_{n f_{i}}\right)\right)
$$

where $\phi$ is the set of edges, $w_{m n}$ is the edge weight between cells $m$ and $n$, and $Z$ is the normalization factor given by:

$$
Z=\sum_{i=1}^{M} \sum_{j=i}^{M}\left(\sum_{(m, n) \in \Phi} w_{m n}\left(\vec{\Omega}_{m f_{i}} \vec{\Omega}_{n f_{j}}+\vec{\Omega}_{m f_{j}} \vec{\Omega}_{n f_{i}}\right)\right)
$$

PMI computation results in values with direct implication to spatial co-occurrence. The PMI values are either negative implicating that a phenotype-pair spatially co-occurs less than the background distribution, positive implicating that a phenotype-pair spatially co-occurs more than the background distribution, or zero implicating that a phenotype-pair spatially co-occurs the same as the background distribution.

\section{Step 3a: Select a background distribution}

We choose to use a random background distribution to depict the probability each CP-pair spatially co-occurs more, less, or the same as random. To construct the random distribution, we 
set the probability of each CP to the probability of each CP over all cells: $p\left(f_{i}^{t}\right)=\frac{1}{N} \sum_{c=1}^{N} \Omega_{c i}$, where $N$ is the total number of cells.

\section{Step 3b: Perform Jackknife estimation}

A jackknife estimation is commonly used to remove bias when measuring the dependence between two random variables (e.g., Pearson's correlation coefficient, mutual information) [6668]. For a given estimator, PMI: $T_{n}=T\left(X_{1}, \ldots, X_{n}\right)$ for $n$ samples. Let $T_{(-i)}$ denote the statistic where the $i$-th patient is removed. The jackknife bias estimate is defined as: $b_{\text {jack }}=$ $(n-1)\left(\bar{T}_{n}-T_{n}\right)$, where $\bar{T}_{n}=\frac{1}{n} \sum_{i} T_{(-i)}$. The bias-corrected estimator is

$$
T_{j a c k}=T_{n}-b_{j a c k}=(n) T_{n}-\left(\frac{n-1}{n}\right) \sum_{i} T_{(-i)}
$$

The jackknife estimate of standard error is: $\widehat{s e}_{j a c k}=\sqrt{\frac{n-1}{n} \sum_{i}\left(T_{(-i)}-\frac{1}{n} \sum_{i} T_{(-i)}\right)^{2}}$.

\section{Step 3c: Filter unstable PMI values}

We observed instability in the PMI estimates (including high jackknife estimated standard error) for any given CP-pair, $\left(f_{i}, f_{j}\right)$ when the number of cells involved in the pairing is low. To remove this bias, we compute the effective number of cells (total ownership probability) in the CP-pair for a patient, $p$, as the summation of ownership probabilities, $N_{\left(f_{i}, f_{j}\right)}^{p}$, over all cells in the network: $N_{\left(f_{i}, f_{j}\right)}^{p}=\sum_{c}^{N^{p}} \vec{\Omega}_{c f_{i}}+\vec{\Omega}_{c f_{j}}$. Aggregating all patients, we use the $25^{\text {th }}$ percentile as a cutoff value and remove all patients with $N_{\left(f_{i}, f_{j}\right)}^{p}<$ cutoff. We find that removing these patients also removes the patients with a relatively high standard error.

Step 4: Identify significant CP-pairs (and microdomains) by comparing PMI distributions $\underline{\text { between recurring and non-recurring cohorts }}$ 
For each CP-pair, $\left(f_{i}, f_{j}\right)$, we compute the Kendall rank correlation, $\tau$, (and p-value, $p_{\tau}$ ) between $\operatorname{PMI}\left(f_{i}, f_{j}\right)$ and time to recurrence (reported in days). In addition, we perform a Wilcoxon ranksum (WRS) test (does not reject the null hypothesis, $h_{W R S}=0$, or does reject the null hypothesis, $h_{W R S}=1$ and corresponding $\mathrm{p}$-value $\left.p_{W R S}\right)$ to determine if the NED-8yrs and REC3yrs $\operatorname{PMI}\left(f_{i}, f_{j}\right)$ values are from equivalent distributions. We define a phenotype pair as significant if $|\tau|>\operatorname{prctile}(\tau, 99 t h), p_{\tau} \leq 0.05, h_{W R S}=1$, and $p_{W R S} \leq 0.05$. Each CP-pair distribution is shown in Figure S9. The Kendall rank correlation and Wilcoxon rank-sum test results are reported for CP-pair (and clinical variables) in Table S4. The 5 CP-pairs identified as significant by our analysis form two microdomains: $\mathcal{M}_{1}$ : CP1-CP1, $\mathcal{M}_{2}$ : CP13-CP9-CP7-CP6CP5.

Step 5: Perform partial correlation analysis to construct outcome-specific biomarker networks $\underline{\text { within each microdomain }}$

For each microdomain, we aim to identify a biomarker network representation for the NED-8yrs and REC-3yrs patient groups. Within a single microdomain and for each patient cohort, we operate on cells with specialized states for the simplicity of network derivation (although ownership probabilities can be incorporated to include transitional and multi-transitional cell states into the network derivation). We then compute the partial correlation between each biomarker-pair, controlling for the remaining confounding biomarkers [1]. First, from the biomarker data $(X: N \times p)$ composed of $N$ cells in the specific microdomain and $p$ biomarkers, we compute the Pearson's correlation coefficient matrix, $C: p \times p$. Second, we invert the correlation coefficient matrix to obtain the precision matrix, $\Phi=C^{-1}$. Third, we compute the partial correlation coefficient between each biomarker pair, $\rho_{i j}$, through normalization: $\rho_{i j}=$ $-\frac{\Phi_{i j}}{\sqrt{\Phi_{i i} \Phi_{j j}}}$. The partial correlation coefficients range from -1 to 1 representing the true correlation between each biomarker pair when all other confounding factors (other biomarkers) 
are removed [37]. For the sake of simplicity, we chose to pool cells across the two patient outcome cohorts: total number of cells in NED-8yrs cohort $=89,963$ and REC-3yrs cohort $=$ 89,596 (Table S3). However, one could also construct patient-specific networks, and based on the number of cells an additional step of matrix regularization may have to be incorporated to avoid overfitting.

To compare the partial correlation networks between the cohorts, NED-8yrs $\left(\rho^{N E D}\right)$ and REC3yrs $\left(\rho^{R E C}\right)$, we use differential connectivity analysis with a permutation test [38]. In particular, we define a symmetric differential connectivity matrix, $\Delta_{0}: p \times p$, where each entry, $(i, j)$, represents the differential connectivity between a biomarker pair: $\Delta_{0}(i, j)=\left|\rho_{i j}^{N E D}-\rho_{i j}^{R E C}\right|$. To test the significance of the differential, we use a permutation test (with $B=10,000$ permutations). For one permutation iteration, the cells from both cohorts are aggregated and randomly sampled to form two data sets, mirroring the same number of cells as the NED-8yrs and REC-3yrs cohorts. Each permutation, $k$, results in a differential connectivity matrix of the permuted data, $\Delta_{k}$. The significance value (p-value) for a specific biomarker-pair $(i, j)$ is computed as $\frac{b+1}{B+1}$ where $b=\sum_{k=1}^{B} I\left(\Delta_{0}(i, j) \leq \Delta_{k}(i, j)\right)$. We define the biomarker-pairs with a differential connectivity score in the top $99^{\text {th }}$ percentile and $p$-value below 0.001 as significant (Table S5, S6). 


\section{Figure captions}

Figure 1 - Unsupervised learning algorithm for cellular phenotypic heterogeneity (LEAPH) on hyperplexed colorectal carcinoma tissue microarray dataset

a. A zoomed in region of a Stage II CRC tissue samples (ca. $0.6 \mathrm{~mm}$ cores). Each subpanel is pseudo-colored with DAPI (blue), and pairs of biomarkers indicated in the subpanel (e.g., subpanel a is pseudo-colored with 4EBP1 (red) and PTEN (green)). We selected a large set of biomarkers $(p=51)$ to test the feasibility of cyclical labeling, imaging, and quenching process of the hyperplexing technology and to provide insights into the CRC biology.

b. LEAPH can perform cellular phenotyping on hyperplexed single sample or a cohort of tissue samples (currently tested up to 213 tissue samples, $500 \mathrm{~K}$ cells). LEAPH applies recursive steps of probabilistic clustering with mixtures of factor analyzer (MFA) models and spatial regularization to construct data-driven computational phenotypic trees. We apply a stopping criterion on the recursion, by applying a threshold on the angle between subspaces created by the mixture models, to ensure that the resulting phenotypes are distinct and to avoid overfitting of the input dataset by LEAPH.

c. The terminal nodes of the tree (leaves) signify distinct computational phenotypes (CP) discovered in the input dataset and form the mixture components of the final MFA model determined by the recursive decomposition. We visualize the spatial distribution of phenotypes within a tissue sample by assigning each cell to the phenotype with the highest ownership probability.

Figure 2 - Illustrating the recursive steps of probabilistic clustering with mixtures of factor analyzer models and spatial regularization in LEAPH

a. Top left: Synthetic data generated to reflect the statistics of the CRC biomarker data. We find that $\mathrm{CRC}$ biomarker values have a wide spread likely due to the individual biomarker response 
sensitivity (e.g., $\log (A L D H 1)$ values range between 0-12, while $\log (C D 20)$ values range between 0-4, see Figure S3). Consequently, we observe dominant phenotypes in the form of epithelial and stromal cells and parsing further we discover the various cell subtypes (e.g., immune, STEM cells). To reflect these properties, the synthetic data has two dominant phenotypes each with two additional subtypes and hence four different phenotypes nested in total. Top right: One tier non-recursive MFA clustering fails at segmenting the four modes in the ground truth synthetic data. Bottom left: Level 1 of the recursive split identifies the two dominant clusters in the synthetic data. Bottom right: Level 2 of the recursive clustering splits each dominant cluster from Level 1 to segment the four clusters from the synthetic data. An example ownership probability vector is shown in each clustering attempt, color coded based on respective cluster. This simulation demonstrates the advantage of performing recursive clustering with MFA rather than a one-tier approach. On the more complex and high-dimensional CRC data, each recursive step of fitting MFA model is repeated 100 times and a consensus model is chosen to eliminate any spurious results that arise from the random cluster initialization.

b. We perform spatial regularization on a single patient sample and track the ownership probabilities for each cell undergoing regularization at each iteration. Majority of cells converge to a specialized cell identity (ownership probabilities $>0.95$ or $<0.05$ ), but a small subset of cells remains in the non-specialized range (ownership probabilities between 0.05 and 0.95 )

c. Tissue sample where each cell is outlined with the ownership probability prior- and postspatial regularization. We specifically point to two cells, each with an ownership probability of 0.8 prior to spatial regularization. Post-regularization, one cell converges to a specialized CP2 (blue) because of the dominance of this CP in its neighborhood (top zoomed box). The second cell remains non-specialized because of the heterogeneity in its neighborhood (bottom zoomed box). 
Figure 3 - LEAPH reveals the hierarchical nature of the cellular phenotypic heterogeneity and identifies specialized, transitional, and rare cell populations in the hyperplexed colorectal carcinoma dataset

a. Cellular phenotypic hierarchy derived by applying LEAPH on the entire CRC cohort. The size of each node is proportional to the fraction of cells with majority ownership to that computational phenotype (CP) (size key). Each leaf node represents a data-driven CP determined by the stopping criteria. The set of all leaf nodes, $13 \mathrm{CPs}$ in total, form the components of the final mixture of factor analyzers model for the CRC patient cohort.

b. For the ease of interpretation, each $\mathrm{CP}$ is characterized by a unique biomarker signature, where the biomarkers are classified as being "ON", "OFF", or "Non-specific (NS)" (see Methods, Figure S7). The biomarker signatures enable identifying heterogeneous populations of known cell types: epithelial cells (CP6, CP7), tumor cells (CP1, CP2, CP3), cancer stem cells (CP5), cancer associated fibroblasts (CP9, CP12), stromal cells (CP4), macrophages (CP8), and immune cells (CP10, CP13).

c. Illustrative tissue samples from clinically relevant patient sub-cohorts. Cell boundaries are outlined with the colors of the assigned phenotype (panel a) based on highest ownership probability values.

d. Example of a neighborhood of non-specialized cells. We point to multiple cells within the neighborhood with varying ownership probabilities. This neighborhood depicts a multitransitional cell state (defined as ownership probabilities spread across more than 2 CPs), transitional cell states (ownership probabilities spread across 2 CPs) compared to a specialized cell state (ownership probability greater than 0.95 for a single CP). 
Figure 4 - Discovery of recurrence-associated microdomains with pointwise mutual information and determine the microdomain-specific network biology

a. Pointwise mutual information (PMI) maps are computed for each tissue sample to quantify the spatial co-occurrence of each CP-pair compared to a random background distribution (see Methods). A PMI value above 0 indicates that the CP-pair spatially co-occurs more often than random and below 0 indicates that the CP-pair spatially co-occurs less often than random. The PMI values are normalized to the range -1 to 1 for visualization.

b. The PMI values for each CP-pair are grouped by the outcome data: no-evidence of recurrence in 8 years (NED-8yrs) and recurrence within 3 years (REC-3yrs). We compared the PMI distributions using the Kendall rank correlation coefficients and the Wilcoxon rank sum test. We found 5 CP-pairs to be significant (see Methods, Table S4, Figure S9). Each significant CPpair has a distribution skewed higher for the REC-3yr group implicating a greater level of spatial co-occurrence compared to a random background distribution.

c. Two microdomains emerge from the significance analysis: microdomain $1\left(\mathcal{M}_{1}-\mathrm{CP} 1:: \mathrm{CP} 1\right)$ consisting of only a single CP and microdomain $2\left(\mathcal{M}_{2}-\mathrm{CP} 13:: \mathrm{CP} 5:: \mathrm{CP} 9:: \mathrm{CP} 6:: \mathrm{CP} 7\right)$ consisting of a network of epithelial and stromal CPs. In the microdomain visualizations, we report the Kendall rank correlation coefficients on each CP-pair edge ( $p$-values for Kendall rank correlation coefficients and Wilcoxon rank sum test are found in Table S4).

d. Within each microdomain, $\mathcal{M}_{1}$ and $\mathcal{M}_{2}$, we identify a partial correlation network for the NED8yrs and REC-3yrs patient cohort consisting of the biomarker pairs with a significant differential in the $99^{\text {th }}$ percentile (see Methods, Table S5 and S6). The biomarkers are grouped based on their presumed cellular functions/processes (Table S2) and the edges connecting each biomarker pair has a color and width corresponding the partial correlation value (also reported in Table S5 and S6). 
Figure 5 - Analytical platform that can be applied to any multi to hyperplexed image data sets.

LEAPH enables the application of a spatial analytics and microdomain-specific network biologybased early discovery and development platform to a multi to hyperplexed imaging platform such as Cell DIVE ${ }^{\text {TM }}$ used in this study, as well as other hyperplexed technologies $[19,20,69]$. The pipeline begins with the preparation of patient tissue samples for pathology including labeling with multi to hyperplexed biomarkers, imaging based on multiple imaging modalities (e.g. transmitted light, fluorescence, and mass spec [20]). Basic image processing and basic image quantitation is then followed by data normalization in preparation of applying the analytical platform. The hyperplexed imaging process can be initiated with a limited set of biomarkers from which LEAPH builds data-driven, computationally unbiased phenotypic hierarchy of cell types, cell states capturing a phenotypic continuum, and their spatial configurations. LEAPH in combination with pointwise mutual information (PMI) discovers outcome-specific microdomains composed of spatially configured computational phenotypes. LEAPH combined with microdomain-specific network biology provides mechanistic insights into disease biology. This analysis will further suggest outcome-specific new pathways, new cellular phenotypes and additional biomarkers which can be optimally tested through iterative probing of the same microdomains on non-destructive imaging platforms such as Cell DIVE ${ }^{\text {TM }}$ [18]. The spatial analytics and microdomain-specific network biology features form the core components of the explainable Al platform [60] that can drive a wide range of applications probing and modulating tumor environment including prognostics, diagnostics, patient stratification for clinical trials, drug target identification, patient-specific therapeutic strategies (e.g. immunotherapy), and animal toxicology studies. 
Figure 1

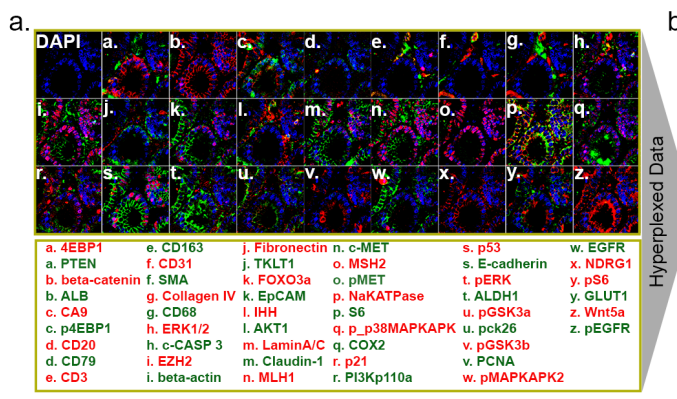

b.
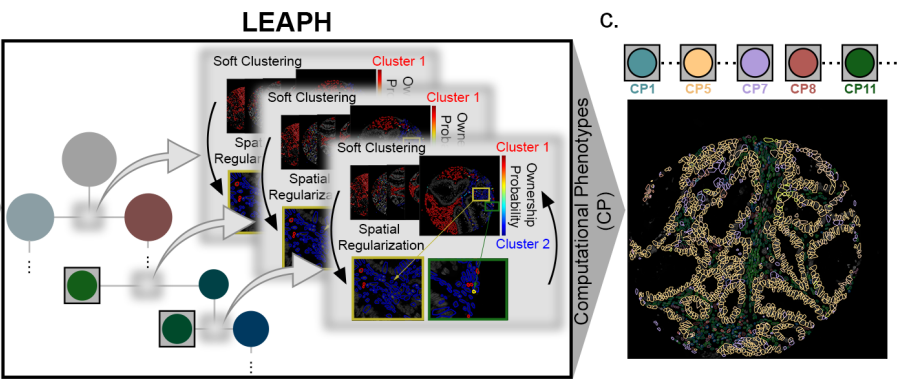

Figure 2
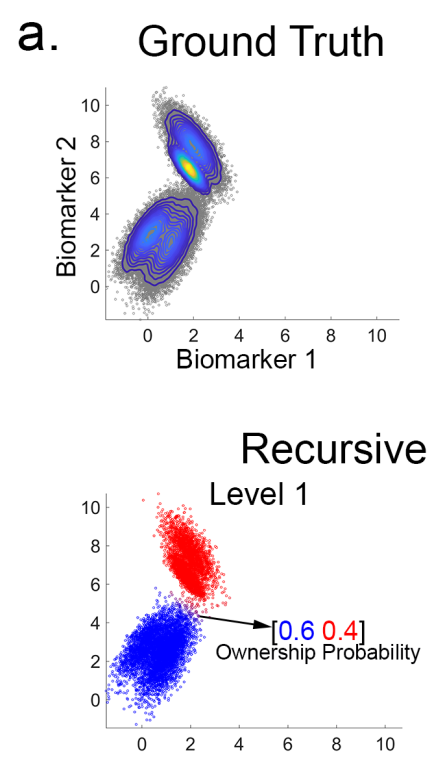

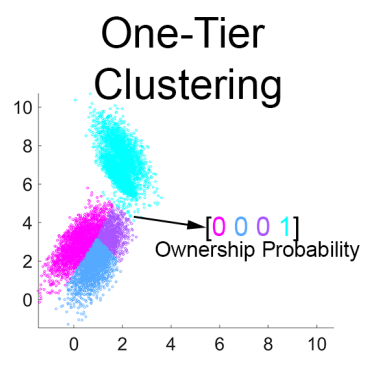

c.

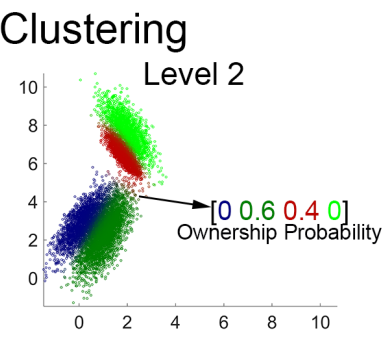

b.
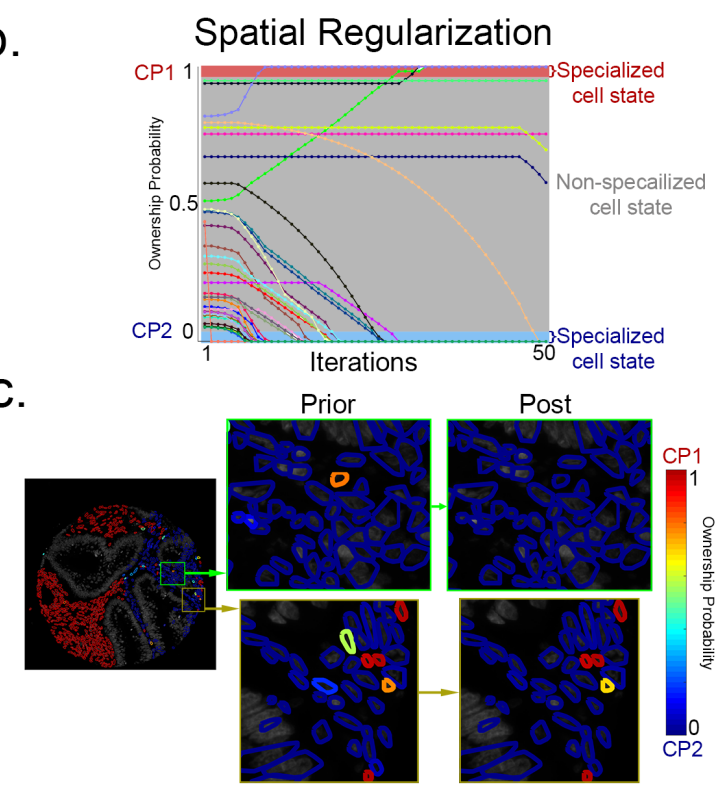
bioRxiv preprint doi: https://doi.org/10.1101/2020.10.02.322529; this version posted October 20, 2020. The copyright holder for this preprint (which was not certified by peer review) is the author/funder. All rights reserved. No reuse allowed without permission.

Figure 3
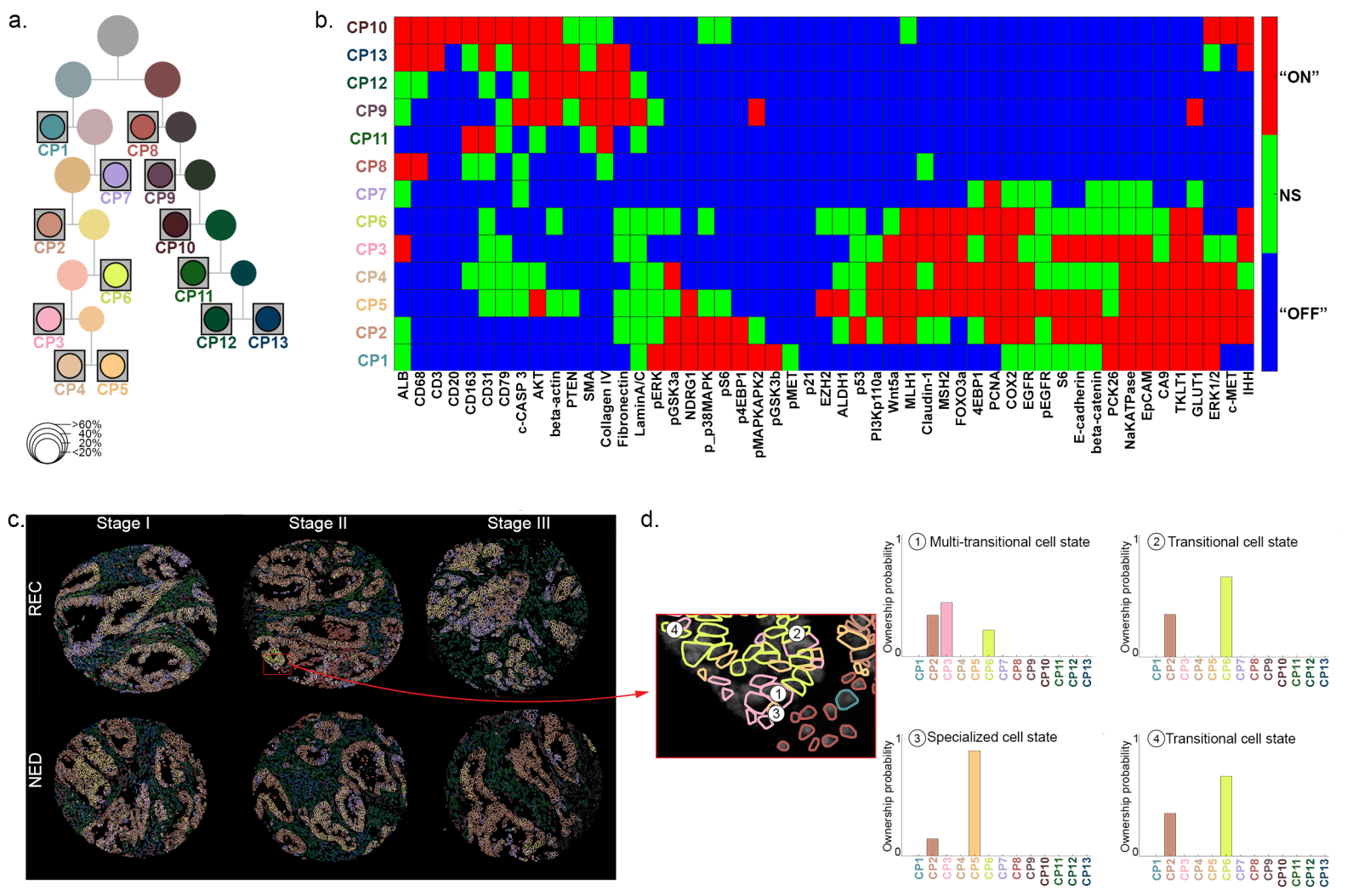
Figure 4
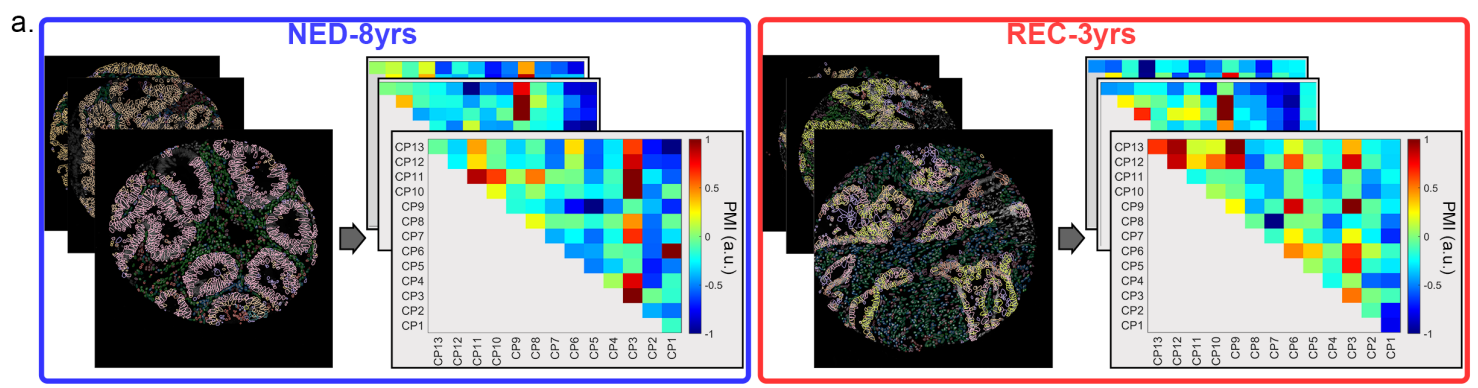

b.
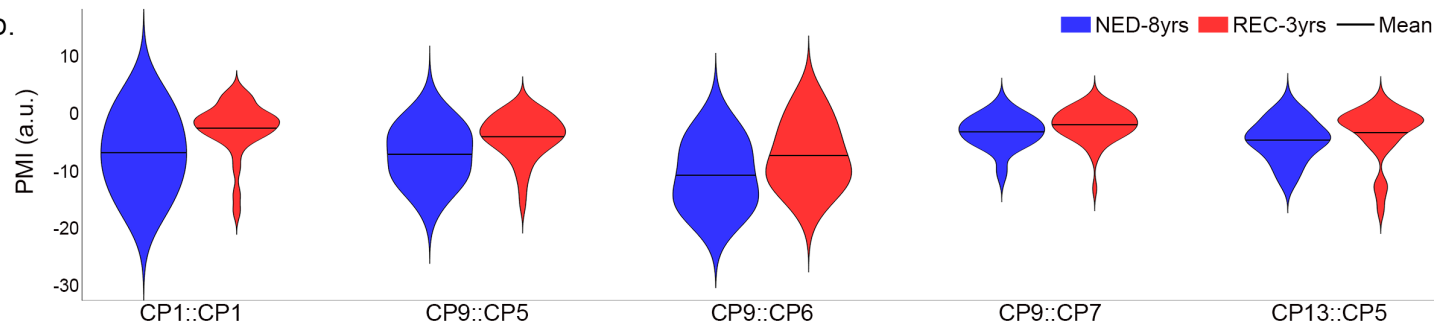

CP1::CP1

CP9::CP5

CP9::CP6

CP9::CP7

CP13::CP5

c.
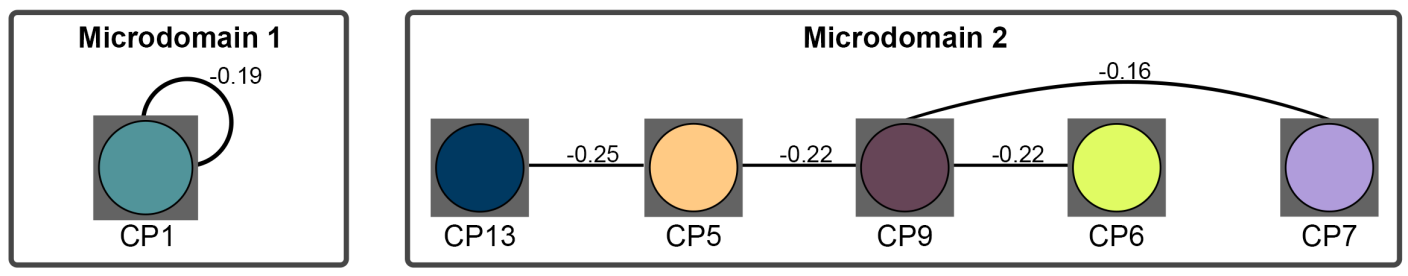

d.

NED-8yrs

REC-3yrs
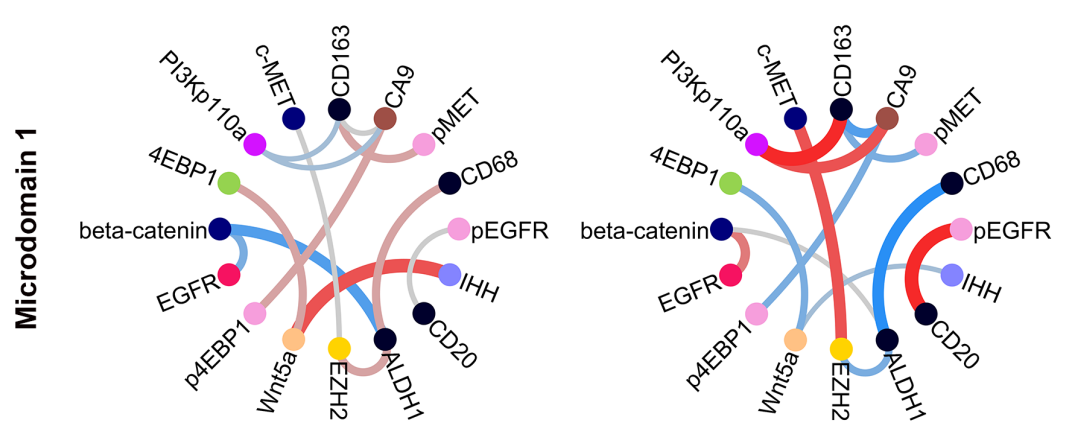

- Adhesion + structure

- Apoptosis

- Cell types + states

- Extracellular transport + metabolism

Hedgehog signaling

Hypoxia signaling

Mismatch repair pathway

- Oncogenic

PI3K/AKT/MTOR pathway

PTM

- RAS/RAF/MAPK pathway

Tumor suppressive

- Adhesion+structure
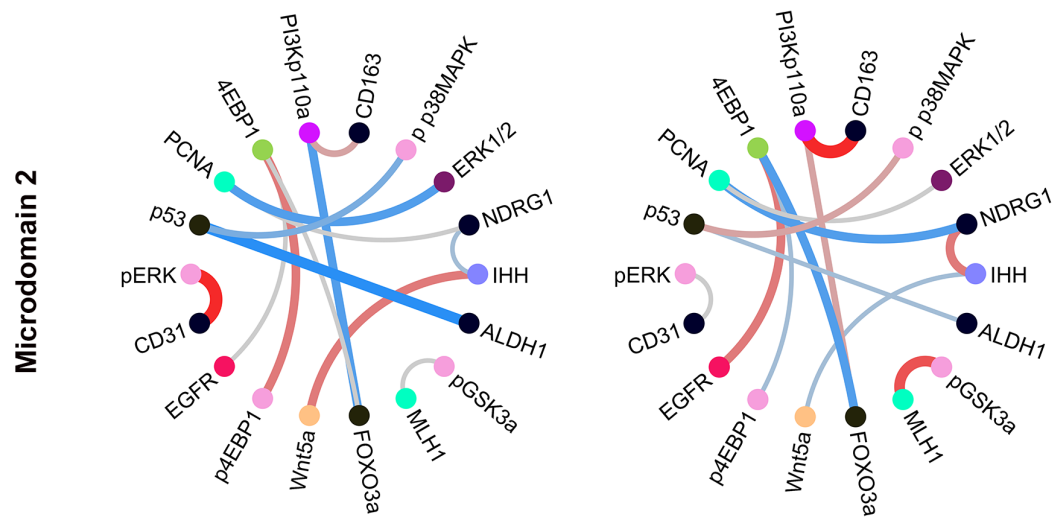

Cell types + states Adhesion + structure Cell types + states Oncogenic

MAPK pathway PISK/AKT/MTOR pathway

Mismatch repair pathway

PIJK/AKT/MTOR pathway

PTM

PIKK/AKT/MTOR pathway

RAS/RAF/MAPK pathway

Tumor suppressive

PI3K/AKT/MTOR pathway

-1 Partial Correlation 
bioRxiv preprint doi: https://doi.org/10.1101/2020.10.02.322529; this version posted October 20, 2020. The copyright holder for this preprint (which was not certified by peer review) is the author/funder. All rights reserved. No reuse allowed without permission.

Figure 5
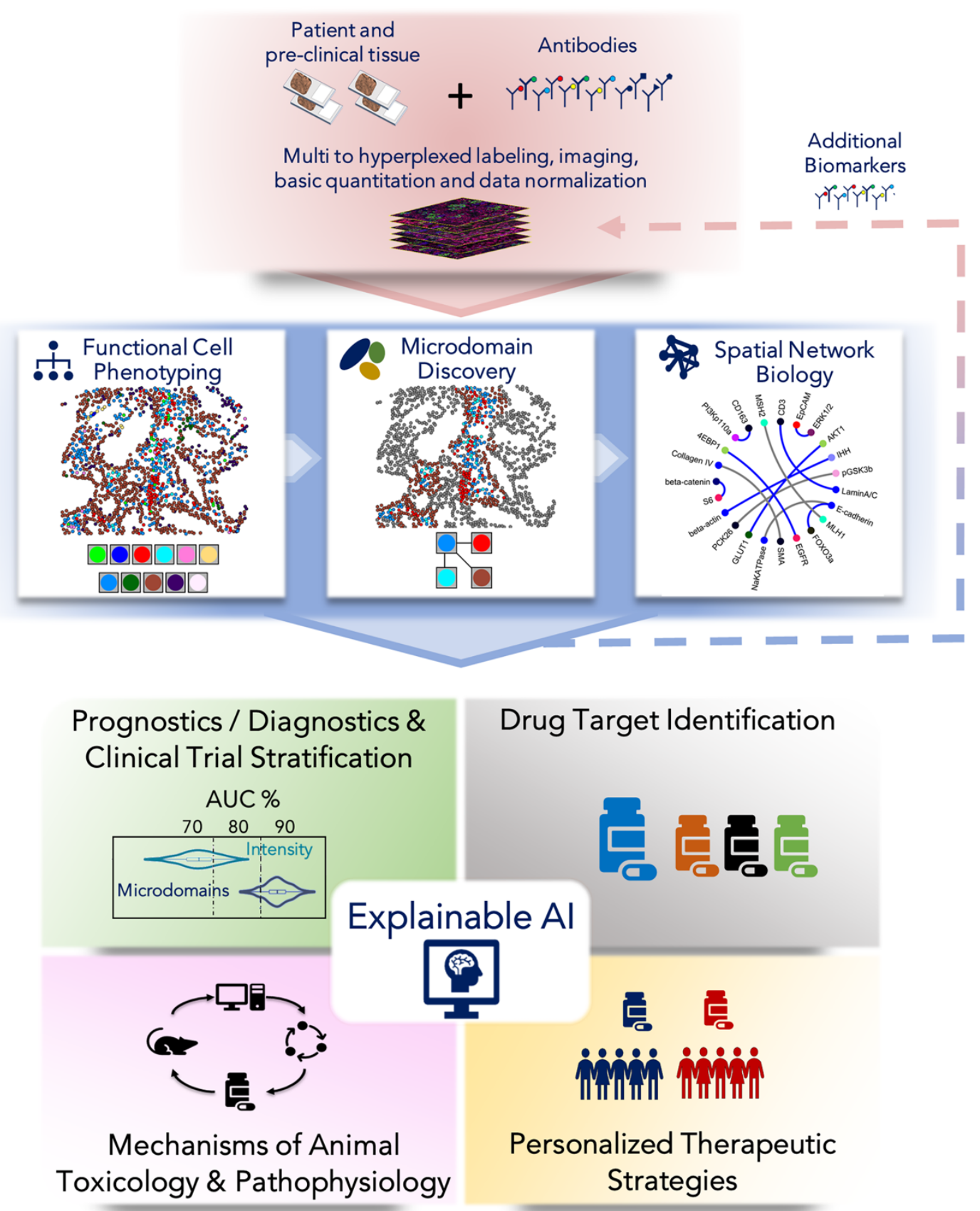


\section{Supplementary Materials for}

\section{Unsupervised cellular phenotypic hierarchy enables spatial intratumor heterogeneity characterization, recurrence-associated microdomains discovery, and harnesses network biology from hyperplexed in-situ fluorescence images of colorectal carcinoma}

Samantha A. Furman ${ }^{1}$, Andrew M. Stern ${ }^{1,2, *}$, Shikhar Uttam ${ }^{1,+}$, D. Lansing Taylor ${ }^{1,2}$, Filippo Pullara $^{3}$, S. Chakra Chennubhotla ${ }^{1,2,3,{ }^{*}}$

${ }^{1}$ Department of Computational and Systems Biology, University of Pittsburgh, PA, 15260, USA

${ }^{2}$ University of Pittsburgh Drug Discovery Institute, University of Pittsburgh, Pittsburgh, PA, 15261, USA

${ }^{3}$ Splntellx, Inc., 2425 Sidney St, Pittsburgh, PA 15203

${ }^{*}$ Corresponding Author Emails: chakra@spintellx.com (S. Chakra Chennubhotla), sternam@pitt.edu (Andrew M. Stern)

This file includes:

Supplementary Table Captions

Supplementary Figure Captions

Supplementary Tables

Supplementary Figures

References

\footnotetext{
+New affiliation: UPMC Hillman Cancer Center, Pittsburgh, PA, 15213, USA
} 


\section{Supplementary Table Captions}

\section{Table S1 - Previous work}

We outline the state-of-the-art cellular phenotyping methods and analysis platforms/studies within the past decade specifying the data investigated, purpose of the study, and integration of the following components: spatial context, soft clustering, hierarchical component, and unsupervised techniques. The data generation methods include the following high-dimensional techniques: mass cytometry (CyTOF) [1], single-cell RNA sequencing (scRNA-seq) [2], multi to hyper - plexed immunofluorescence imaging (MxIF/HxIF) [3, 4], imaging mass cytometry (IMC) [5], small molecule fluorescent in situ hybridization (smFISH) [6], and flow cytometry [7]. Many single-cell clustering algorithms are supervised methods subjected to user-defined number of clusters and/or approximated ground truth reliant on prior biological knowledge [8-11]. Popular methods, PhenoGraph [12] and SPADE [13], dissect cell clusters from CyTOF data but lack a spatial component. All the analysis methods listed assume each cell belongs to exactly one celltype (hard-clustering) leaving no room to identify cell states along a cellular phenotypic continuum (transitional and multi-transitional cell states). To our knowledge, there does not exist a cellular phenotyping method with a combined spatial analysis framework which is unsupervised, harnesses a spatial component, and promotes the identification of the cellular phenotypic continuum.

\section{Table S2 - CRC biomarkers}

List of the biomarkers in the CRC hyperplexed data set. Grayed biomarkers are eliminated due to quality control.

\section{Table S3 - CRC patient statistics}

Patient statistics for the clinical data of the selected tissue samples. 
Table S4 - Correlation of pointwise mutual information with time-to-recurrence

Correlation analysis with the clinical information (sex, grade, stage, age), CP fractions per tissue sample, and CP-pair pointwise mutual information (PMI) values. We compute the Kendall rank correlation coefficient, $\tau$, (and significance, $p_{\tau}$ ) between each covariate and the time-torecurrence (days). In addition, we perform the Wilcoxon rank-sum test (hypothesis $h_{W R S}$ and significance, $p_{W R S}$ ) to determine if for each covariate, the NED-8yrs and REC-3yrs patient tissue samples originate from the same underlying distribution. Covariates are considered significant (highlighted in yellow) if $h_{W R S}=1\left(p_{W R S} \leq 0.05\right)$ and $|\tau|>0.1\left(p_{\tau} \leq 0.05\right)$.

Table S5 - Microdomain $1\left(\mathcal{M}_{1}\right)$ specific biomarker interaction networks with partial correlation analysis

Within each spatial microdomain $\left(\mathcal{M}_{1}: \mathrm{CP} 1:: \mathrm{CP} 1, \mathcal{M}_{2}: \mathrm{CP} 13:: \mathrm{CP} 9:: \mathrm{CP} 7:: \mathrm{CP} 6:: \mathrm{CP} 5\right)$ we compute the partial correlation network (1,275 pairs total) for the NED-8yrs and REC-3yrs patient groups and compute the differential $\left(\Delta_{0}\right)$ between the cohorts with a corresponding $p$ value using a permutation test (see Methods). The partial correlation values represent the true correlation between two biomarkers when all other confounding factors (other biomarkers) are removed. This table reports the biomarker pairs with a significant differential in the $99^{\text {th }}$ percentile within $\mathcal{M}_{1}$

Table S6 - Microdomain $2\left(\mathcal{M}_{2}\right)$ specific biomarker interaction networks with partial correlation analysis

With the same method as described in Table S5, we report the biomarker pairs with a significant differential in the $99^{\text {th }}$ percentile within microdomain $2\left(\mathcal{M}_{2}:\right.$ CP13::CP9::CP7::CP6::CP5).

\section{Table S7 - Biomarker order for virtual simulation}


Biomarkers listed in order based on discriminative ranking for LEAPH performed on ALL-DATA

(Figure 4). The epithelial-stromal dissection is from level 1 of the LEAPH hierarchy and the epithelial/stromal sub-dissection is from level 2. Each ranking is used to determine the order of adding biomarkers to LEAPH to reveal the minimal subset of biomarkers needed to reproduce the original computational phenotypes (Figure S10). 


\section{Supplementary Figure Captions}

\section{Figure S1 - Cell segmentation}

a. Pseudo colored image of a tissue sample with DAPI (blue), E-cadherin (red), NaKATPase (green, top), and SMA (green, bottom) biomarkers used for cell segmentation.

b. The raw cell segmentation masks (top) and the smoothed cell masks used for visualization (bottom). The zoomed in regions of the raw and smoothed cell masks show a closer visual of the cell borders.

\section{Figure S2 - Biomarker functions and properties}

List of the biomarkers in the CRC hyperplexed data set organized by their assumed cellular functions, or involvement in cellular processes, derived from the literature (see Table S1 for more information). Bolded biomarkers hold more than one cellular function (see legend in gray box).

\section{Figure S3 - Cell count quality control}

Tissue damage and other imaging artifacts impact biomarker staining and subsequent cell segmentation. We observe this variability in the number of cells segmented from each spot, shown here as a histogram. We remove all spots with less than a set 1000 cell threshold based on the $20^{\text {th }}$ percentile of the distribution (gray box).

\section{Figure S4 - Non-normal distribution of log transformed biomarker values}

Histograms of biomarker values over $\sim 500 \mathrm{~K}$ cells from 213 tissue samples. The $\mathrm{x}$-axis is the biomarker values log transformed which range between 0 and 12. Each biomarker has a dynamic range of values, e.g., $\log (A L D H 1): 0-12$ but $\log (C D 20): 0-4$. The probability range (yaxis) is same across all plots (0-0.12). The values alongside each biomarker distribution represent the kurtosis. When compared to a Gaussian distribution (kurtosis=3), many of the 
biomarker distributions in our data are non-Gaussian with kurtosis values above (superGaussian) and below 3 (sub-Gaussian).

\section{Figure S5 - Variance for each biomarker distribution}

Variance for each biomarker over the entire CRC data. We chose a two-dimensional latent space for the MFA model, as we observed this is enough to capture the input variance.

\section{Figure S6 - Mixture of Factor Analyzers (MFA) model selection}

MFA model parameters are learned through the Expectation-Maximization (EM) algorithm with a random initialization and provides no guarantee to converge to a global minimum [14]. To account for this and ensure stability, we perform a hundred different EM optimizations, each initialized randomly. Each optimization yields an MFA model with a set of model parameters. We compute the biomarker ranking for each set of model parameters (see Methods) and aggregate all biomarker rankings to compute their mean ranking. The model with a biomarker ranking closest (Euclidean distance) to the mean ranking is selected as the consensus model and deemed to provide an optimal subspace representation.

\section{Figure S7 - CP-specific biomarker classifications}

Each CP is characterized with a unique biomarker signature (Figure $3 \mathrm{~b}$ ). The signatures are obtained by comparing the histogram of biomarker values over all cells (Figure S4) vs the histogram of biomarker values over the specialized cells in each CP (see Methods). The figure panel shows the cumulative distribution functions (CDFs) for all biomarkers (gray lines) and the $20^{\text {th }}$ and $80^{\text {th }}$ percentiles (see legend) in each CDF. The biomarker distribution over all specialized cells in each CP is represented by the mean (diamonds color-coordinated with Figure 4) and an error bar showing the $20^{\text {th }}$ and $80^{\text {th }}$ percentile values. Each biomarker is classified as "ON", "OFF”, "NS" (see Methods for more details). 


\section{Figure S8 caption}

The biomarker signatures are computed to facilitate qualitative interpretation only. The signatures shown in Figure $3 \mathrm{~b}$ is an interpretation of each $\mathrm{CP}$ as a constellation of biomarkers based on the total CRC cohort of 213 patients. After reducing the patient cohort to the NED-8yrs and REC-3yrs sub-groups (91 patients), we re-interpret the CPs by computing the biomarker signatures (see Methods) based on only the reduced patient cohort (a).

Further, we compute the CP biomarker signatures separately for the (b) NED-3yrs (45 patients) and (c) REC-8yrs (46 patients) cohorts. To compute the cohort-specific biomarker signatures, we are specifically comparing the biomarker distributions of each CP within each cohort compared to the biomarker distribution based on all cells in the reduced patient cohort (91 patients).

\section{Figure S9 - Distribution of PMI values}

The distributions of PMI values for each CP-pair grouped by the outcome data (NED-8yrs in blue, REC-3yrs in red). Comparing the distributions using the Kendall rank correlation coefficients and the Wilcoxon rank sum test, we found 5 significant CP-pairs highlighted in yellow (see Methods and Table S4).

\section{Figure S10 - What is the minimal set of biomarkers needed to reproduce the LEAPH results with 51 biomarkers?}

The cyclical process of labeling, imaging, and quenching of the hyperplexing imaging platforms permits selecting biomarkers on demand. Because LEAPH determines an ordered list of key biomarkers at each level of the hierarchy, we performed a virtual simulation where the tissue is assumed to be labeled with a subset of the key biomarkers (as opposed to the 51 biomarkers that we started with). The key biomarkers are selected based on the order of discriminative biomarkers from the ALL-DATA results with 51 biomarkers (Table S7). LEAPH is then run to 
determine if the phenotype identities for each cell changed from the ones obtained with 51 biomarkers. For comparing phenotyping identities, we use the maximum CP ownership probability from the ALL-DATA results with 51 biomarkers (Figure 3) as the ground truth label for each cell. We measure the accuracy as the percentage of cells with a matching CP identity to the ALL-DATA results with 51 biomarkers. We ran this experiment to test the phenotypic identities at first two levels of the hierarchy: epithelial and stromal division at the first level and their individual subtypes at the second.

a. As expected, the epithelial-stromal dissection is low-dimensional, only requiring 2 biomarkers (pck26, E-cadherin) to obtain an accuracy above 97\%. Increasing the number of biomarkers further increases the accuracy to almost $99 \%$.

b-c. Both subtype dissections require a larger set of biomarkers than the first level. The stromal subtype dissection (b) reaches above 93\% accuracy with 4 biomarkers (Lamin A/C, Claudin 1, Akt, 4EBP1). The addition of more biomarkers leads to an accuracy above $97 \%$ with 8 or more biomarkers. The epithelial subtype dissection (c) reaches $89 \%$ accuracy with 4 biomarkers (4EBP1, pS6, pMAPKAPK2, PCNA) but an increase in the number of biomarkers does not make a substantial difference in the reproduction accuracy. 


\section{References}

1. Bandura, D.R., et al., Mass cytometry: technique for real time single cell multitarget immunoassay based on inductively coupled plasma time-of-flight mass spectrometry. Analytical chemistry, 2009. 81(16): p. 6813-6822.

2. Tang, F., et al., mRNA-Seq whole-transcriptome analysis of a single cell. Nature methods, 2009. 6(5): p. 377-382.

3. Gerdes, M.J., et al., Highly multiplexed single-cell analysis of formalin-fixed, paraffinembedded cancer tissue. Proc Natl Acad Sci U S A, 2013. 110(29): p. 11982-7.

4. Goltsev, Y., et al., Deep Profiling of Mouse Splenic Architecture with CODEX Multiplexed Imaging. Cell, 2018. 174(4): p. 968-981 e15.

5. Giesen, C., et al., Highly multiplexed imaging of tumor tissues with subcellular resolution by mass cytometry. Nature methods, 2014. 11(4): p. 417-422.

6. Raj, A., et al., Imaging individual mRNA molecules using multiple singly labeled probes. Nature methods, 2008. 5(10): p. 877-879.

7. Chattopadhyay, P.K., et al., Quantum dot semiconductor nanocrystals for immunophenotyping by polychromatic flow cytometry. Nature medicine, 2006. 12(8): p. $972-977$.

8. Chen, W.S., et al., Uncovering axes of variation among single-cell cancer specimens. Nature methods, 2020.

9. Chen, Z., et al., Modeling Multiplexed Images with Spatial-LDA Reveals Novel Tissue Microenvironments. Journal of Computational Biology, 2020.

10. Zhu, Q., et al., Identification of spatially associated subpopulations by combining scRNAseq and sequential fluorescence in situ hybridization data. Nat Biotechnol, 2018. 
11. Santamaria-Pang, A., et al. Robust single cell quantification of immune cell subtypes in histological samples. in 2017 IEEE EMBS International Conference on Biomedical \& Health Informatics (BHI). IEEE.

12. Levine, Jacob H., et al., Data-Driven Phenotypic Dissection of AML Reveals Progenitorlike Cells that Correlate with Prognosis. Cell, 2015. 162(1): p. 184-197.

13. Qiu, P., et al., Extracting a cellular hierarchy from high-dimensional cytometry data with SPADE. Nature biotechnology, 2011. 29(10): p. 886.

14. Ghahramani, Z. and G.E. Hinton, The EM algorithm for mixtures of factor analyzers. 1996, Technical Report CRG-TR-96-1, University of Toronto.

15. Jackson, H.W., et al., The single-cell pathology landscape of breast cancer. Nature, 2020: p. 1-6.

16. Sun, Z., et al., A Bayesian mixture model for clustering droplet-based single-cell transcriptomic data from population studies. Nat Commun, 2019. 10(1): p. 1649.

17. McKinley, E.T., et al., Optimized multiplex immunofluorescence single-cell analysis reveals tuft cell heterogeneity. $\mathrm{JCl}$ insight, 2017. 2(11).

18. Samusik, N., et al., Automated mapping of phenotype space with single-cell data. Nature methods, 2016. 13(6): p. 493-496.

19. Chen, H., et al., Cytofkit: A Bioconductor Package for an Integrated Mass Cytometry Data Analysis Pipeline. PLoS computational biology, 2016. 12(9): p. e1005112.

20. Amir, E.-a.D., et al., viSNE enables visualization of high dimensional single-cell data and reveals phenotypic heterogeneity of leukemia. Nature biotechnology, 2013. 31(6): p. $545-552$.

21. Francipane, M.G. and E. Lagasse, mTOR pathway in colorectal cancer: an update. Oncotarget, 2014. 5(1): p. 49. 
22. González-Trejo, S., et al., Baseline serum albumin and other common clinical markers are prognostic factors in colorectal carcinoma: a retrospective cohort study. Medicine, 2017. 96(15).

23. Fujikawa, H., et al., Prognostic impact of preoperative albumin-to-globulin ratio in patients with colon cancer undergoing surgery with curative intent. Anticancer research, 2017. 37(3): p. 1335-1342.

24. Huang, H., et al., Validation of Prognosis Value of Cumulative Prognostic Scores Based on Serum High-Density Lipoprotein Cholesterol and Albumin Levels in Patients with Colorectal Cancer. Journal of Cancer, 2019. 10(1): p. 35.

25. Watson, A., Apoptosis and colorectal cancer. Gut, 2004. 53(11): p. 1701-1709.

26. Greenhough, A., et al., Cancer cell adaptation to hypoxia involves a HIF-GPRC5A-YAP axis. EMBO molecular medicine, 2018. 10(11).

27. Burotto, M., et al., The MAPK pathway across different malignancies: a new perspective. Cancer, 2014. 120(22): p. 3446-3456.

28. Farhan, M., et al., FOXO signaling pathways as therapeutic targets in cancer. International journal of biological sciences, 2017. 13(7): p. 815.

29. Bullock, M., et al., FOXO3 expression during colorectal cancer progression: biomarker potential reflects a tumour suppressor role. British journal of cancer, 2013. 109(2): p. 387-394.

30. Feng, W., et al., Role of glucose metabolism related gene GLUT1 in the occurrence and prognosis of colorectal cancer. Oncotarget, 2017. 8(34): p. 56850.

31. Shen, Y.-M., et al., Overexpression of GLUT1 in colorectal cancer is independently associated with poor prognosis. The International journal of biological markers, 2011. 26(3): p. 166-172.

32. Wu, C., et al., Hedgehog signaling pathway in colorectal cancer: function, mechanism, and therapy. OncoTargets and therapy, 2017. 10: p. 3249. 
33. Li, G.-M., Mechanisms and functions of DNA mismatch repair. Cell research, 2008. 18(1): p. 85-98.

34. Li, X.-L., et al., P53 mutations in colorectal cancer-molecular pathogenesis and pharmacological reactivation. World journal of gastroenterology: WJG, 2015. 21(1): p.

\section{4.}

35. Molinari, F. and M. Frattini, Functions and regulation of the PTEN gene in colorectal cancer. Frontiers in oncology, 2014. 3: p. 326.

36. Ahopelto, K., et al., Transketolase-like protein 1 expression predicts poor prognosis in colorectal cancer. Cancer biology \& therapy, 2016. 17(2): p. 163-168.

37. Langbein, S., et al., Expression of transketolase TKTL1 predicts colon and urothelial cancer patient survival: Warburg effect reinterpreted. British journal of cancer, 2006. 94(4): p. 578-585.

38. Ying, J., et al., WNT5A exhibits tumor-suppressive activity through antagonizing the Wnt/ $\beta$-catenin signaling, and is frequently methylated in colorectal cancer. Clinical cancer research, 2008. 14(1): p. 55-61.

39. Pan, S., et al., Decreased expression of ARHGAP15 promotes the development of colorectal cancer through PTEN/AKT/FOXO1 axis. Cell death \& disease, 2018. 9(6): p. $1-13$. 


\section{Supplementary Tables}

\section{Table S1 - previous work}

\begin{tabular}{|c|c|c|c|c|c|c|c|}
\hline Previous method & Year & Input data & Purpose & $\begin{array}{l}\text { Spatial } \\
\text { context }\end{array}$ & Soft clustering & $\begin{array}{l}\text { Hierarchical } \\
\text { component }\end{array}$ & Unsupervised \\
\hline $\begin{array}{l}\text { Jackson, H.W., et al. } \\
\text { [15] }\end{array}$ & 2020 & IMC & $\begin{array}{l}\text { Spatially resolved, single-cell } \\
\text { analysis to characterize } \\
\text { intratumor phenotypic } \\
\text { heterogeneity }\end{array}$ & Yes & No & No & No \\
\hline Spatial-LDA [9] & 2020 & MxIF/HxIF & $\begin{array}{l}\text { Recover microenvironment } \\
\text { signatures }\end{array}$ & Yes & No & No & No \\
\hline PhMED [8] & 2020 & $\begin{array}{l}\text { CyTOF, } \\
\text { scRNA-seq }\end{array}$ & $\begin{array}{l}\text { Embedding a 'manifold of } \\
\text { manifolds' }\end{array}$ & No & No & Yes & No \\
\hline BAMM-SC [16] & 2019 & scRNA-seq & $\begin{array}{l}\text { Cluster scRNA-seq data from } \\
\text { multiple individuals } \\
\text { simultaneously }\end{array}$ & No & No & No & Yes \\
\hline Zhu, Q., et al. [10] & 2018 & $\begin{array}{l}\text { scRNAseq, } \\
\text { smFISH }\end{array}$ & $\begin{array}{l}\text { Distinguish between intrinsic } \\
\text { and extrinsic effects of global } \\
\text { gene expression }\end{array}$ & Yes & No & No & No \\
\hline $\begin{array}{l}\text { McKinley, E.T., et al. } \\
\text { [17] }\end{array}$ & 2017 & HxIF & $\begin{array}{l}\text { Quantify tuft cell number and } \\
\text { distribution throughout the } \\
\text { mouse small intestine and } \\
\text { colon }\end{array}$ & Yes & No & No & No \\
\hline $\begin{array}{l}\text { Santamaria-Pang, A., } \\
\text { et al. [11] }\end{array}$ & 2017 & $\mathrm{HxIF}$ & $\begin{array}{l}\text { Machine-learning based } \\
\text { method for classifying immune } \\
\text { cell types in human tissue }\end{array}$ & No & No & Yes & No \\
\hline X-shift [18] & 2016 & СуTOF & $\begin{array}{l}\text { Automated cell-subset } \\
\text { clustering }\end{array}$ & No & No & No & Yes \\
\hline Cytofkit [19] & 2016 & СуTOF & $\begin{array}{l}\text { Platform integrating } \\
\text { bioinformatics methods and in- } \\
\text { house novel algorithms for } \\
\text { cytometry data analysis }\end{array}$ & No & No & No & No \\
\hline PhenoGraph [12] & 2015 & СуTOF & $\begin{array}{l}\text { Algorithmically defines } \\
\text { phenotypes in high- } \\
\text { dimensional single cell data }\end{array}$ & No & No & No & Yes \\
\hline viSNE [20] & 2013 & CyTOF & $\begin{array}{l}\text { Tool to map high-dimensional } \\
\text { data onto two dimensions }\end{array}$ & No & No & No & No \\
\hline SPADE [13] & 2011 & $\begin{array}{l}\text { Flow } \\
\text { cytometry, } \\
\text { CyTOF }\end{array}$ & $\begin{array}{l}\text { Extract a hierarchy of cellular } \\
\text { heterogeneity }\end{array}$ & No & No & Yes & Yes \\
\hline
\end{tabular}

Table S2 - CRC biomarkers

\begin{tabular}{|l|l|l|}
\hline Biomarker & Formal Name & Cellular Function \\
\hline 4EBP1 & $\begin{array}{l}\text { Eukaryotic translation initiation factor } \\
\text { 4Ebinding protein 1 }\end{array}$ & $\begin{array}{l}\text { Signaling pathways - } \\
\text { PI3K/AKT/MTOR [21] }\end{array}$ \\
\hline AKT1 & RAC-alpha serine/threonine-protein kinase & $\begin{array}{l}\text { Signaling pathways - } \\
\text { PI3K/AKT/MTOR [21] }\end{array}$ \\
\hline ALB & Albumin & $\begin{array}{l}\text { Extracellular transport } \\
\text { and metabolism [22-24] }\end{array}$ \\
\hline ALDH1 & $\begin{array}{l}\text { Aldehyde dehydrogenase 1 family, } \\
\text { member A1 }\end{array}$ & $\begin{array}{l}\text { Cell types and states - } \\
\text { STEM }\end{array}$ \\
\hline beta-actin & one of six actin isoforms & Adhesion and structure \\
\hline beta-catenin & Catenin beta-1 & Oncogenic \\
\hline c-CASP 3 & Cleaved caspase 3 & $\begin{array}{l}\text { Signaling pathways - } \\
\text { apoptosis [25] }\end{array}$ \\
\hline c-MET & $\begin{array}{l}\text { Tyrosine-protein kinase Met; also known } \\
\text { as hepatocyte growth factor receptor }\end{array}$ & Oncogenic \\
\hline
\end{tabular}




\begin{tabular}{|c|c|c|}
\hline CA9 & Carbonic anhydrase 9 & $\begin{array}{l}\text { Signaling pathways - } \\
\text { hypoxia signaling [26] }\end{array}$ \\
\hline CD163 & $\begin{array}{l}\text { Scavenger receptor cysteine-rich type } 1 \\
\text { protein M130; macrophage marker }\end{array}$ & $\begin{array}{l}\text { Cell types and states - } \\
\text { macrophages }\end{array}$ \\
\hline CD20 & B-cell differentiation antigen & $\begin{array}{l}\text { Cell types and states - } \\
\text { B cells }\end{array}$ \\
\hline CD3 & T-cell surface glycoprotein CD3 delta chai & $\begin{array}{l}\text { Cell types and states - } \\
\text { T cells }\end{array}$ \\
\hline CD31 & Platelet endothelial cell adhesion molecule & $\begin{array}{l}\text { Cell types and states - } \\
\text { Endothelial cells }\end{array}$ \\
\hline CD68 & $\begin{array}{l}\text { Transmembrane glycoprotein macrophage } \\
\text { marker }\end{array}$ & $\begin{array}{l}\text { Cell types and states - } \\
\text { macrophages }\end{array}$ \\
\hline CD79 & $\begin{array}{l}\text { B-cell antigen receptor complex-associated } \\
\text { protein alpha chain }\end{array}$ & $\begin{array}{l}\text { Cell types and states - } \\
\text { B cells }\end{array}$ \\
\hline Claudin-1 & Transmembrane tight junction protein & Adhesion and structure \\
\hline Collagen IV & $\begin{array}{l}\text { Collagen IV - Structural component of } \\
\text { glomerular basement membranes }\end{array}$ & Adhesion and structure \\
\hline COX2 & Cyclooxygenase-2 & Oncogenic \\
\hline E-cadherin & $\begin{array}{l}\text { Epithelial calcium-dependent adhesion } \\
\text { molecule }\end{array}$ & Adhesion and structure \\
\hline EGFR & Epidermal growth factor receptor & $\begin{array}{l}\text { Signaling pathways - } \\
\text { PI3K/AKT/MTOR [21], } \\
\text { RAS/RAF/MEK [27] }\end{array}$ \\
\hline EpCAM & Epithelial Cell Adhesion Molecule & $\begin{array}{l}\text { Oncogenic } \\
\text { Adhesion and structure }\end{array}$ \\
\hline ERK1/2 & Extracellular signal-regulated kinases & $\begin{array}{l}\text { Signaling pathways - } \\
\text { RAS/RAF/MEK [27] }\end{array}$ \\
\hline $\mathrm{EZH} 2$ & Enhancer of zeste homolog 2 & $\begin{array}{l}\text { Signaling pathways - } \\
\text { PI3K/AKT/MTOR [21], } \\
\text { RAS/RAF/MEK [27] } \\
\text { Oncogenic } \\
\text { Cell types and states - } \\
\text { tumor cell }\end{array}$ \\
\hline Fibronectin & Extracellular matrix glycoprotein & $\begin{array}{l}\text { Adhesion and structure } \\
\text { Cell types and states }\end{array}$ \\
\hline FOXO3a & Forkhead box protein $\mathrm{O}$ & $\begin{array}{l}\text { Signaling pathways - } \\
\text { PI3K/AKT/MTOR [21] } \\
\text { Tumor suppressive [28, } \\
\text { 29] }\end{array}$ \\
\hline GLUT1 & Glucose transporter 1 & $\begin{array}{l}\text { Extracellular transport } \\
\text { and metabolism }[30, \\
31]\end{array}$ \\
\hline $\mathrm{IHH}$ & Indian hedgehog protein & $\begin{array}{l}\text { Signaling pathways - } \\
\text { hedgehog signaling [32] }\end{array}$ \\
\hline LaminA/C & nuclear lamina protein & Adhesion and structure \\
\hline MLH1 & MutL homolog 1 & $\begin{array}{l}\text { Signaling pathways - } \\
\text { mismatch repair [33] }\end{array}$ \\
\hline MSH2 & MutS protein homolog 2 & $\begin{array}{l}\text { Signaling pathways - } \\
\text { mismatch repair [33] }\end{array}$ \\
\hline
\end{tabular}




\begin{tabular}{|c|c|c|}
\hline NaKATPase & $\begin{array}{l}\text { Sodium-potassium pump; } \\
\text { transmembrane ATPase enzyme }\end{array}$ & Adhesion and structure \\
\hline NDRG1 & N-myc downstream-regulated gene 1 & Cell types and states \\
\hline $\mathrm{p} 21$ & Cyclin-dependent kinase inhibitor & $\begin{array}{l}\text { Signaling pathways - } \\
\text { PI3K/AKT/MTOR [21], } \\
\text { mismatch repair [33] }\end{array}$ \\
\hline p4EBP1 & & $\begin{array}{l}\text { Phosphorylated } \\
\text { proteins }\end{array}$ \\
\hline p53 & Tumor protein & $\begin{array}{l}\text { Signaling pathways - } \\
\text { PI3K/AKT/MTOR [21] } \\
\text { Tumor suppressive [34] }\end{array}$ \\
\hline PCK26 & Pan cytokeratin & $\begin{array}{l}\text { Cell types and states - } \\
\text { tumor cells }\end{array}$ \\
\hline PCNA & Proliferating cell nuclear antigen & $\begin{array}{l}\text { Signaling pathways - } \\
\text { mismatch repair [33] }\end{array}$ \\
\hline pEGFR & $\begin{array}{l}\text { Phosphorylated epidermal growth factor } \\
\text { receptor }\end{array}$ & $\begin{array}{l}\text { Phosphorylated } \\
\text { proteins }\end{array}$ \\
\hline pERK & $\begin{array}{l}\text { Phosphorylated extracellular signal- } \\
\text { regulated kinases }\end{array}$ & $\begin{array}{l}\text { Phosphorylated } \\
\text { proteins }\end{array}$ \\
\hline pGSK3a & & $\begin{array}{l}\text { Phosphorylated } \\
\text { proteins }\end{array}$ \\
\hline pGSK3b & & $\begin{array}{l}\text { Phosphorylated } \\
\text { proteins }\end{array}$ \\
\hline PI3Kp110a & $\begin{array}{l}\text { Phosphatidylinositol-4, 5-bisphosphate 3- } \\
\text { kinase, catalytic subunit alpha }\end{array}$ & $\begin{array}{l}\text { Signaling pathways - } \\
\text { PI3K/AKT/MTOR [21] }\end{array}$ \\
\hline pMAPKAPK2 & & $\begin{array}{l}\text { Phosphorylated } \\
\text { proteins }\end{array}$ \\
\hline pMET & & $\begin{array}{l}\text { Phosphorylated } \\
\text { proteins }\end{array}$ \\
\hline pS6 & Phosphorylated ribosomal protein S6 & $\begin{array}{l}\text { Phosphorylated } \\
\text { proteins }\end{array}$ \\
\hline PTEN & Phosphatase and tensin homolog & Tumor suppressive [35] \\
\hline p_p38MAPK & & $\begin{array}{l}\text { Phosphorylated } \\
\text { proteins }\end{array}$ \\
\hline S6 & Ribosomal protein S6 & $\begin{array}{l}\text { Signaling pathways - } \\
\text { PI3K/AKT/MTOR [21], } \\
\text { RAS/RAF/MEK [27] }\end{array}$ \\
\hline SMA & alpha smooth muscle actin & $\begin{array}{l}\text { Cell types and states - } \\
\text { cancer associated } \\
\text { fibroblasts (CAFs) }\end{array}$ \\
\hline TKLT1 & Transketolase-like protein 1 & $\begin{array}{l}\text { Extracellular transport } \\
\text { and metabolism [36, } \\
37]\end{array}$ \\
\hline Wnt $5 a$ & $\begin{array}{l}\text { Ligand for members of the frizzled family of } \\
\text { seven transmembrane receptors }\end{array}$ & Tumor suppressive [38] \\
\hline CD8 & $\begin{array}{l}\text { T-cell surface glycoprotein CD8 alpha } \\
\text { chain }\end{array}$ & $\begin{array}{l}\text { Cell types and states - } \\
\mathrm{T} \text { cells }\end{array}$ \\
\hline CK19 & Keratin, type I cytoskeletal 19 & Adhesion and structure \\
\hline FOXO1 & Forkhead box protein $\mathrm{O} 1$ & $\begin{array}{l}\text { Signaling pathways - } \\
\text { PI3K/AKT/MTOR [21] }\end{array}$ \\
\hline
\end{tabular}




\begin{tabular}{|l|l|l|}
\hline & & $\begin{array}{l}\text { Tumor suppressive [28, } \\
\text { 39] }\end{array}$ \\
\hline pNDRG1 & $\begin{array}{l}\text { Phosphorylated n-myc downstream- } \\
\text { regulated gene 1 }\end{array}$ & $\begin{array}{l}\text { Phosphorylated } \\
\text { proteins }\end{array}$ \\
\hline
\end{tabular}

Table S3 - CRC patient statistics

\begin{tabular}{|c|c|c|c|c|c|}
\hline DATA & All data & NED-5yrs & REC-5yrs & NED-8yrs & REC-3yrs \\
\hline $\begin{array}{l}\text { \# patients } \\
(\%)\end{array}$ & $\begin{array}{l}213 \\
(100 \%)\end{array}$ & $\begin{array}{l}154 \\
(72.3 \%)\end{array}$ & $\begin{array}{l}59 \\
(27.7 \%)\end{array}$ & $\begin{array}{l}45 \\
(21.1 \%)\end{array}$ & $\begin{array}{l}46 \\
(21.6 \%)\end{array}$ \\
\hline $\begin{array}{l}\text { \# males } \\
(\%)\end{array}$ & $\begin{array}{l}105 \\
(49.3 \%)\end{array}$ & $\begin{array}{l}73 \\
(34.3 \%)\end{array}$ & $\begin{array}{l}32 \\
(15 \%)\end{array}$ & $\begin{array}{l}15 \\
(7 \%) \\
\end{array}$ & $\begin{array}{l}28 \\
(13.1 \%)\end{array}$ \\
\hline $\begin{array}{l}\text { Mean age } \\
\text { (std) }\end{array}$ & $\begin{array}{l}63.6 \\
(7.2) \\
\end{array}$ & $\begin{array}{l}63 \\
(7.1) \\
\end{array}$ & $\begin{array}{l}65.4 \\
(7.2) \\
\end{array}$ & $\begin{array}{l}62.8 \\
(6.4) \\
\end{array}$ & $\begin{array}{l}65.4 \\
(7.2) \\
\end{array}$ \\
\hline $\begin{array}{l}\text { Mean time- } \\
\text { to-recurrence } \\
\text { (std) }\end{array}$ & $\begin{array}{l}1922.3 \\
(1142.1)\end{array}$ & $\begin{array}{l}2393.3 \\
(951.2)\end{array}$ & $\begin{array}{l}692.7 \\
(504.4)\end{array}$ & $\begin{array}{l}3582.8 \\
(469.3)\end{array}$ & $\begin{array}{l}464.6 \\
(278.1)\end{array}$ \\
\hline $\begin{array}{l}\text { \# Stage I } \\
(\%)\end{array}$ & $\begin{array}{l}94 \\
(44.1 \%)\end{array}$ & $\begin{array}{l}91 \\
(42.7 \%)\end{array}$ & $\begin{array}{l}3 \\
(1.4 \%)\end{array}$ & $\begin{array}{l}25 \\
(11.7 \%)\end{array}$ & $\begin{array}{l}2 \\
(0.9 \%)\end{array}$ \\
\hline $\begin{array}{l}\text { \# Stage II } \\
(\%)\end{array}$ & $\begin{array}{l}75 \\
(35.2 \%)\end{array}$ & $\begin{array}{l}55 \\
(25.8 \%)\end{array}$ & $\begin{array}{l}20 \\
(9.4 \%)\end{array}$ & $\begin{array}{l}18 \\
(8.5 \%)\end{array}$ & $\begin{array}{l}15 \\
(7 \%)\end{array}$ \\
\hline $\begin{array}{l}\text { \# Stage III } \\
(\%)\end{array}$ & $\begin{array}{l}44 \\
(20.7 \%)\end{array}$ & $\begin{array}{l}8 \\
(3.8 \%)\end{array}$ & $\begin{array}{l}36 \\
(16.9 \%)\end{array}$ & $\begin{array}{l}2 \\
(0.9 \%)\end{array}$ & $\begin{array}{l}29 \\
(13.6 \%)\end{array}$ \\
\hline $\begin{array}{l}\text { Mean \# cells } \\
\text { per tissue } \\
\text { sample } \\
\text { (std) }\end{array}$ & $\begin{array}{l}2082.7 \\
(513.4)\end{array}$ & $\begin{array}{l}2103 \\
(492.3)\end{array}$ & $\begin{array}{l}2029.9 \\
(565.7)\end{array}$ & $\begin{array}{l}1999.2 \\
(476.8)\end{array}$ & $\begin{array}{c}1947.7 \\
(593.9)\end{array}$ \\
\hline $\begin{array}{l}\text { Total \# of } \\
\text { cells }\end{array}$ & 443618 & 323856 & 119762 & 89963 & 89596 \\
\hline
\end{tabular}

Table S4 - PMI correlation analysis

\begin{tabular}{|l|l|l|l|l|l|l|}
\hline Covariate & $\begin{array}{l}\text { Correlation } \\
\text { coefficient } \\
(\text { Kendall } \\
\text { rank, } \boldsymbol{\tau})\end{array}$ & $\begin{array}{l}\text { Correlation } \\
\text { coefficient } \\
\text { significance } \\
\left(\boldsymbol{p}_{\boldsymbol{\tau}}\right)\end{array}$ & $\begin{array}{l}\text { Wilcoxon } \\
\text { rank-sum } \\
\text { test } \\
\left(\boldsymbol{h}_{\text {WRS }}\right)\end{array}$ & $\begin{array}{l}\text { Wilcoxon } \\
\text { rank-sum } \\
\text { test } \\
\text { significance } \\
\left(\boldsymbol{p}_{\text {WRS }}\right)\end{array}$ & $\begin{array}{l}\text { \# NED- } \\
\mathbf{8 y r s}\end{array}$ & $\begin{array}{l}\text { \# REC- } \\
\text { 3yrs }\end{array}$ \\
\hline stage & -0.449 & 0 & 0 & 1 & 45 & 46 \\
\hline grade & -0.377 & 0 & 0 & 1 & 45 & 45 \\
\hline CP13::CP5 & -0.251 & 0.0029 & 0.0362 & 1 & 35 & 31 \\
\hline CP9::CP6 & -0.216 & 0.0161 & 0.0302 & 1 & 32 & 27 \\
\hline CP9::CP5 & -0.215 & 0.0051 & 0.005 & 1 & 40 & 39 \\
\hline sex & 0.207 & 0.0169 & 0.009 & 1 & 45 & 46 \\
\hline CP1::CP1 & -0.188 & 0.0376 & 0.014 & 1 & 29 & 29 \\
\hline CP13::CP6 & -0.183 & 0.0572 & 0.0446 & 1 & 29 & 23 \\
\hline CP9::CP7 & -0.159 & 0.0259 & 0.0198 & 1 & 45 & 46 \\
\hline CP5::CP1 & -0.148 & 0.0668 & 0.0211 & 1 & 34 & 38 \\
\hline CP7::CP6 & -0.14 & 0.0903 & 0.0426 & 1 & 37 & 32 \\
\hline CP8::CP4 & 0.139 & 0.1181 & 0.0475 & 1 & 31 & 29 \\
\hline
\end{tabular}




\begin{tabular}{|c|c|c|c|c|c|c|}
\hline CP12::CP6 & -0.124 & 0.1512 & 0.0337 & 1 & 32 & 31 \\
\hline CP4::CP2 & 0.053 & 0.5042 & 0.0236 & 1 & 36 & 39 \\
\hline CP12::CP5 & -0.245 & 0.0018 & 0.069 & 0 & 39 & 37 \\
\hline CP12::CP3 & -0.205 & 0.011 & 0.0519 & 0 & 39 & 33 \\
\hline CP13::CP3 & -0.188 & 0.0219 & 0.0703 & 0 & 38 & 32 \\
\hline CP5::CP5 & -0.161 & 0.1079 & 0.1349 & 0 & 28 & 20 \\
\hline CP13::CP13 & 0.159 & 0.0441 & 0.0556 & 0 & 39 & 36 \\
\hline CP7::CP5 & -0.15 & 0.0515 & 0.1481 & 0 & 39 & 40 \\
\hline age & -0.148 & 0.0427 & 0.0558 & 0 & 45 & 46 \\
\hline CP9::CP3 & -0.147 & 0.0727 & 0.315 & 0 & 36 & 34 \\
\hline CP5::CP2 & -0.146 & 0.0588 & 0.2096 & 0 & 40 & 38 \\
\hline CP10::CP5 & -0.146 & 0.0814 & 0.0626 & 0 & 34 & 33 \\
\hline CP13::CP8 & 0.144 & 0.0486 & 0.489 & 0 & 43 & 44 \\
\hline CP7::CP3 & -0.137 & 0.0873 & 0.2389 & 0 & 39 & 34 \\
\hline CP8 & 0.133 & 0.0632 & 0.3979 & 0 & 45 & 46 \\
\hline CP5 & -0.132 & 0.0656 & 0.248 & 0 & 45 & 46 \\
\hline CP6::CP2 & -0.127 & 0.1208 & 0.1183 & 0 & 38 & 32 \\
\hline CP11::CP5 & -0.125 & 0.1283 & 0.1485 & 0 & 35 & 35 \\
\hline CP8::CP5 & -0.124 & 0.1512 & 0.0973 & 0 & 33 & 30 \\
\hline CP13 & 0.117 & 0.1013 & 0.2547 & 0 & 45 & 46 \\
\hline CP13::CP11 & 0.115 & 0.112 & 0.3541 & 0 & 45 & 43 \\
\hline CP11::CP4 & 0.112 & 0.1924 & 0.3034 & 0 & 34 & 30 \\
\hline CP11::CP3 & -0.111 & 0.1798 & 0.5444 & 0 & 35 & 34 \\
\hline CP9::CP9 & -0.11 & 0.1719 & 0.2111 & 0 & 37 & 35 \\
\hline CP8::CP7 & 0.109 & 0.1377 & 0.3872 & 0 & 41 & 45 \\
\hline CP6::CP3 & 0.108 & 0.2506 & 0.2682 & 0 & 27 & 27 \\
\hline CP6::CP5 & -0.106 & 0.2431 & 0.0836 & 0 & 31 & 27 \\
\hline CP3::CP3 & -0.103 & 0.2761 & 0.2181 & 0 & 25 & 29 \\
\hline CP11::CP9 & -0.101 & 0.1616 & 0.0512 & 0 & 43 & 46 \\
\hline CP5::CP3 & -0.099 & 0.2458 & 0.4745 & 0 & 33 & 32 \\
\hline CP9 & -0.096 & 0.179 & 0.0857 & 0 & 45 & 46 \\
\hline CP2::CP1 & -0.095 & 0.1919 & 0.2679 & 0 & 43 & 44 \\
\hline CP8::CP3 & -0.094 & 0.2873 & 0.4483 & 0 & 32 & 29 \\
\hline CP10::CP6 & -0.094 & 0.3016 & 0.1345 & 0 & 31 & 27 \\
\hline CP10::CP8 & 0.093 & 0.2235 & 0.9231 & 0 & 37 & 43 \\
\hline CP10::CP3 & -0.092 & 0.2838 & 0.7524 & 0 & 32 & 32 \\
\hline CP11::CP7 & -0.09 & 0.2135 & 0.3187 & 0 & 44 & 45 \\
\hline CP11::CP10 & 0.089 & 0.224 & 0.554 & 0 & 42 & 44 \\
\hline CP12::CP8 & 0.089 & 0.2188 & 0.3391 & 0 & 44 & 45 \\
\hline CP10::CP4 & 0.084 & 0.3194 & 0.051 & 0 & 35 & 32 \\
\hline CP13::CP12 & 0.081 & 0.2654 & 0.4602 & 0 & 44 & 44 \\
\hline CP11::CP6 & -0.077 & 0.3818 & 0.2568 & 0 & 32 & 30 \\
\hline CP3 & -0.071 & 0.3183 & 0.7298 & 0 & 45 & 46 \\
\hline CP10::CP9 & -0.069 & 0.3404 & 0.1161 & 0 & 44 & 45 \\
\hline CP3::CP2 & -0.069 & 0.3848 & 0.787 & 0 & 38 & 37 \\
\hline CP8::CP6 & -0.069 & 0.5096 & 0.2337 & 0 & 25 & 21 \\
\hline CP4 & 0.069 & 0.3387 & 0.0734 & 0 & 45 & 46 \\
\hline CP13::CP4 & 0.065 & 0.4365 & 0.2406 & 0 & 36 & 32 \\
\hline CP9::CP2 & -0.065 & 0.3685 & 0.3835 & 0 & 45 & 45 \\
\hline
\end{tabular}




\begin{tabular}{|c|c|c|c|c|c|c|}
\hline CP12::CP7 & -0.063 & 0.3853 & 0.2842 & 0 & 45 & 44 \\
\hline CP8::CP2 & 0.063 & 0.4019 & 0.1206 & 0 & 42 & 42 \\
\hline CP7::CP1 & -0.062 & 0.3874 & 0.1232 & 0 & 45 & 45 \\
\hline CP6::CP6 & -0.061 & 0.5999 & 0.4053 & 0 & 24 & 14 \\
\hline CP13::CP2 & 0.061 & 0.401 & 0.2891 & 0 & 45 & 43 \\
\hline CP4::CP1 & -0.059 & 0.4467 & 0.6284 & 0 & 39 & 38 \\
\hline CP11::CP1 & -0.059 & 0.4276 & 0.0975 & 0 & 42 & 43 \\
\hline CP8::CP1 & 0.057 & 0.4755 & 0.9789 & 0 & 37 & 38 \\
\hline CP12::CP4 & 0.054 & 0.5099 & 0.4209 & 0 & 36 & 34 \\
\hline CP7::CP4 & 0.054 & 0.4984 & 0.2092 & 0 & 38 & 37 \\
\hline CP10 & 0.054 & 0.4507 & 0.9588 & 0 & 45 & 46 \\
\hline CP7::CP2 & -0.053 & 0.4632 & 0.8793 & 0 & 45 & 44 \\
\hline CP8::CP8 & 0.052 & 0.5617 & 0.5247 & 0 & 29 & 31 \\
\hline CP11::CP8 & 0.051 & 0.4938 & 0.7325 & 0 & 40 & 43 \\
\hline CP13::CP10 & 0.05 & 0.5039 & 0.7678 & 0 & 41 & 43 \\
\hline CP9::CP1 & -0.05 & 0.5088 & 0.1943 & 0 & 42 & 41 \\
\hline CP1 & -0.05 & 0.4886 & 0.0871 & 0 & 45 & 46 \\
\hline CP12::CP9 & -0.049 & 0.499 & 0.4338 & 0 & 45 & 45 \\
\hline CP6 & -0.048 & 0.4993 & 0.821 & 0 & 45 & 46 \\
\hline CP3::CP1 & -0.047 & 0.58 & 0.4152 & 0 & 32 & 34 \\
\hline CP6::CP1 & -0.047 & 0.6224 & 0.7488 & 0 & 28 & 26 \\
\hline CP7 & -0.045 & 0.5282 & 0.921 & 0 & 45 & 46 \\
\hline CP4::CP4 & 0.045 & 0.6515 & 0.2989 & 0 & 24 & 26 \\
\hline CP12::CP1 & -0.041 & 0.5809 & 0.1746 & 0 & 41 & 45 \\
\hline CP11 & 0.041 & 0.5693 & 0.9273 & 0 & 45 & 46 \\
\hline CP9::CP8 & 0.04 & 0.5813 & 0.6048 & 0 & 43 & 45 \\
\hline CP10::CP10 & 0.037 & 0.6441 & 0.6156 & 0 & 37 & 36 \\
\hline CP6::CP4 & -0.035 & 0.6885 & 0.3542 & 0 & 34 & 28 \\
\hline CP7::CP7 & -0.035 & 0.6569 & 0.9148 & 0 & 39 & 38 \\
\hline CP5::CP4 & -0.034 & 0.6961 & 0.6987 & 0 & 33 & 32 \\
\hline CP9::CP4 & -0.031 & 0.7008 & 0.795 & 0 & 39 & 36 \\
\hline CP13::CP7 & 0.03 & 0.6758 & 0.6283 & 0 & 45 & 45 \\
\hline CP11::CP2 & -0.03 & 0.6838 & 0.6642 & 0 & 45 & 43 \\
\hline CP11::CP11 & 0.027 & 0.7353 & 0.555 & 0 & 36 & 37 \\
\hline CP2 & 0.027 & 0.7112 & 0.2716 & 0 & 45 & 46 \\
\hline CP12::CP12 & -0.026 & 0.743 & 0.8651 & 0 & 39 & 39 \\
\hline CP12::CP10 & 0.025 & 0.7304 & 0.9155 & 0 & 44 & 43 \\
\hline CP4::CP3 & 0.025 & 0.7735 & 0.6628 & 0 & 33 & 33 \\
\hline CP12::CP11 & -0.021 & 0.7687 & 0.86 & 0 & 44 & 45 \\
\hline CP2::CP2 & 0.021 & 0.8035 & 0.8825 & 0 & 31 & 37 \\
\hline CP12 & -0.016 & 0.8263 & 0.8707 & 0 & 45 & 46 \\
\hline CP13::CP1 & -0.013 & 0.862 & 0.4338 & 0 & 42 & 42 \\
\hline CP10::CP2 & 0.01 & 0.8882 & 0.701 & 0 & 45 & 43 \\
\hline CP10::CP1 & -0.006 & 0.9382 & 0.2922 & 0 & 40 & 41 \\
\hline CP10::CP7 & 0.005 & 0.9463 & 0.8535 & 0 & 45 & 44 \\
\hline CP12::CP2 & -0.005 & 0.9474 & 0.6805 & 0 & 44 & 43 \\
\hline CP13::CP9 & 0.003 & 0.9684 & 0.7291 & 0 & 44 & 44 \\
\hline
\end{tabular}


Table S5 - $\mathcal{M}_{1}$ specific network biology

\begin{tabular}{|l|l|l|l|l|}
\hline Biomarker-pair & $\begin{array}{l}\text { NED-8yrs } \\
\text { partial } \\
\text { correlation }\end{array}$ & $\begin{array}{l}\text { REC-3yrs } \\
\text { partial } \\
\text { correlation }\end{array}$ & \multicolumn{1}{|c|}{$\mathbf{\Delta}_{\mathbf{0}}$} & p-value $\left.\mathbf{(}_{\mathbf{0}}\right)$ \\
\hline ALDH1::CD68 & 0.137 & -0.378 & 0.515 & 0.0001 \\
\hline CD163::PI3Kp110a & -0.026 & 0.428 & 0.454 & 0.0001 \\
\hline Wnt5a::IHH & 0.337 & -0.057 & 0.394 & 0.0001 \\
\hline pMET::CD163 & 0.165 & -0.191 & 0.357 & 0.0001 \\
\hline EZH2::ALDH1 & 0.163 & -0.192 & 0.355 & 0.0001 \\
\hline CA9::PI3Kp110a & -0.044 & 0.308 & 0.353 & 0.0001 \\
\hline CD20::pEGFR & 0.082 & 0.425 & 0.344 & 0.0001 \\
\hline p4EBP1::CA9 & 0.135 & -0.195 & 0.33 & 0.0001 \\
\hline $\begin{array}{l}\text { beta- } \\
\text { catenin::EGFR }\end{array}$ & -0.11 & 0.206 & 0.316 & 0.0001 \\
\hline CA9::CD163 & 0.051 & -0.256 & 0.308 & 0.0001 \\
\hline EZH2::C-MET & 0.089 & 0.395 & 0.306 & 0.0001 \\
\hline $\begin{array}{l}\text { beta- } \\
\text { catenin::ALDH1 }\end{array}$ & -0.227 & 0.071 & 0.298 & 0.0001 \\
\hline Wnt5a::4EBP1 & 0.137 & -0.155 & 0.293 & 0.0001 \\
\hline
\end{tabular}

Table S6 - $\mathcal{M}_{2}$ specific network biology

\begin{tabular}{|l|l|l|l|l|}
\hline Biomarker-pair & $\begin{array}{l}\text { NED-8yrs } \\
\text { partial } \\
\text { correlation }\end{array}$ & $\begin{array}{l}\text { REC-3yrs } \\
\text { partial } \\
\text { correlation }\end{array}$ & \multicolumn{1}{|c|}{$\mathbf{\Delta}_{\mathbf{0}}$} & p-value $\left(\boldsymbol{\Delta}_{\mathbf{0}}\right)$ \\
\hline pERK::CD31 & 0.426 & 0.034 & 0.392 & 0.0001 \\
\hline FOXO3a::PI3Kp110a & -0.209 & 0.111 & 0.32 & 0.0001 \\
\hline Wnt5a::IHH & 0.203 & -0.098 & 0.301 & 0.0001 \\
\hline MLH1::pGSK3a & 0.035 & 0.323 & 0.288 & 0.0001 \\
\hline IHH::NDRG1 & -0.022 & 0.265 & 0.287 & 0.0001 \\
\hline NDRG1::PCNA & 0.049 & -0.232 & 0.28 & 0.0001 \\
\hline ALDH1::p53 & -0.347 & -0.067 & 0.28 & 0.0001 \\
\hline p_p38MAPK::p53 & -0.112 & 0.159 & 0.271 & 0.0001 \\
\hline p4EBP1::4EBP1 & 0.263 & -0.007 & 0.27 & 0.0001 \\
\hline EGFR::4EBP1 & 0.006 & 0.274 & 0.267 & 0.0001 \\
\hline ERK1/2::PCNA & -0.219 & 0.039 & 0.258 & 0.0001 \\
\hline CD163::PI3Kp110a & 0.165 & 0.421 & 0.256 & 0.0001 \\
\hline FOXO3a::4EBP1 & 0.041 & -0.213 & 0.255 & 0.0001 \\
\hline
\end{tabular}

Table S7 - biomarker order for virtual simulation

\begin{tabular}{|l|l|l|l|}
\hline & $\begin{array}{l}\text { Epithelial-stromal } \\
\text { dissection }\end{array}$ & Stromal sub-dissection & Epithelial sub-dissection \\
\hline 1 & pck26 & Lamin A/C & 4EBP1 \\
\hline 2 & E-cadherin & Claudin 1 & pS6 \\
\hline 3 & NaKATPase & Akt & pMAPKAPK2 \\
\hline 4 & SMA & 4EBP1 & PCNA \\
\hline 5 & Beta Catenin & CD79 & pERK \\
\hline
\end{tabular}




\begin{tabular}{|c|c|c|c|}
\hline 6 & 4EBP1 & pl3Kp110a & pGSK3beta \\
\hline 7 & S6 & SMA & Claudin1 \\
\hline 8 & Claudin1 & CD163 & pl3Kp110a \\
\hline 9 & COX2 & PCNA & p4EBP1 \\
\hline 10 & PCNA & FOXO3a & pck26 \\
\hline 11 & $\mathrm{EZH} 2$ & ALDH1 & $\mathrm{MSH} 2$ \\
\hline 12 & EPCAM & Fibronectin & $\mathrm{EZH} 2$ \\
\hline 13 & CA9 & pGSK3a & CD163 \\
\hline 14 & CollV & NDRG1 & P53 \\
\hline 15 & p53 & MLH1 & MLH1 \\
\hline 16 & MSH2 & Beta Actin & p p38MAPK \\
\hline 17 & Wnt5 & ERK & Beta Catenin \\
\hline 18 & pGSK3beta & S6 & CD79 \\
\hline 19 & EGFR & pERK & Foxo 3a \\
\hline 20 & Beta Actin & PTEN & S6 \\
\hline 21 & NDRG1 & Wnt5 & Akt \\
\hline 22 & Foxo $3 a$ & pMAPKAPK2 & Wnt5 \\
\hline 23 & MLH1 & Beta Catenin & Albumin \\
\hline 24 & TKLP1 & Col IV & pMET \\
\hline 25 & pS6 & Pck26 & NaKATPase \\
\hline 26 & Lamin A/C & $\mathrm{IHH}$ & COX2 \\
\hline 27 & Fibronectin & $\mathrm{MSH} 2$ & CD68 \\
\hline 28 & Glut1 & Albumin & pGSK3a \\
\hline 29 & pGSK3alpha & CD68 & TKLP1 \\
\hline 30 & CD68 & CD3 & ALDH1 \\
\hline 31 & p4EBP1 & EGFR & $\mathrm{IHH}$ \\
\hline 32 & p21 & TKLP1 & NDRG1 \\
\hline 33 & $\mathrm{IHH}$ & CA9 & CA9 \\
\hline 34 & ERK & CD31 & EGFR \\
\hline 35 & pl3Kp110a & EPCAM & EPCAM \\
\hline 36 & PTEN & p53 & PTEN \\
\hline 37 & CD31 & Glut1 & CD31 \\
\hline 38 & pp38MAPK & p p38MAPK & Beta Actin \\
\hline 39 & CD3 & p21 & Lamin A/C \\
\hline 40 & Akt & cMET epitomics & cMET epitomics \\
\hline 41 & cMET epitomics & CD20 & E-cadherin \\
\hline 42 & Albumin & pS6 & SMA \\
\hline 43 & Cleaved caspase 3 & p4EBP1 & Fibronectin \\
\hline 44 & pEGFR & NaKATPase & CD3 \\
\hline 45 & pMAPKAPK2 & COX2 & Glut1 \\
\hline 46 & CD163 & $\mathrm{EZH} 2$ & p21 \\
\hline 47 & ALDH1 & Cleaved Caspase 3 & CD20 \\
\hline 48 & pERK & E-cadherin & CollV \\
\hline 49 & CD79 & pMET & pEGFR \\
\hline 50 & CD20 & pEGFR & ERK \\
\hline 51 & pMET & pGSK3beta & Cleaved Caspase 3 \\
\hline
\end{tabular}


bioRxiv preprint doi: https://doi.org/10.1101/2020.10.02.322529; this version posted October 20, 2020. The copyright holder for this preprint (which was not certified by peer review) is the author/funder. All rights reserved. No reuse allowed without permission.

\section{Figure S1}
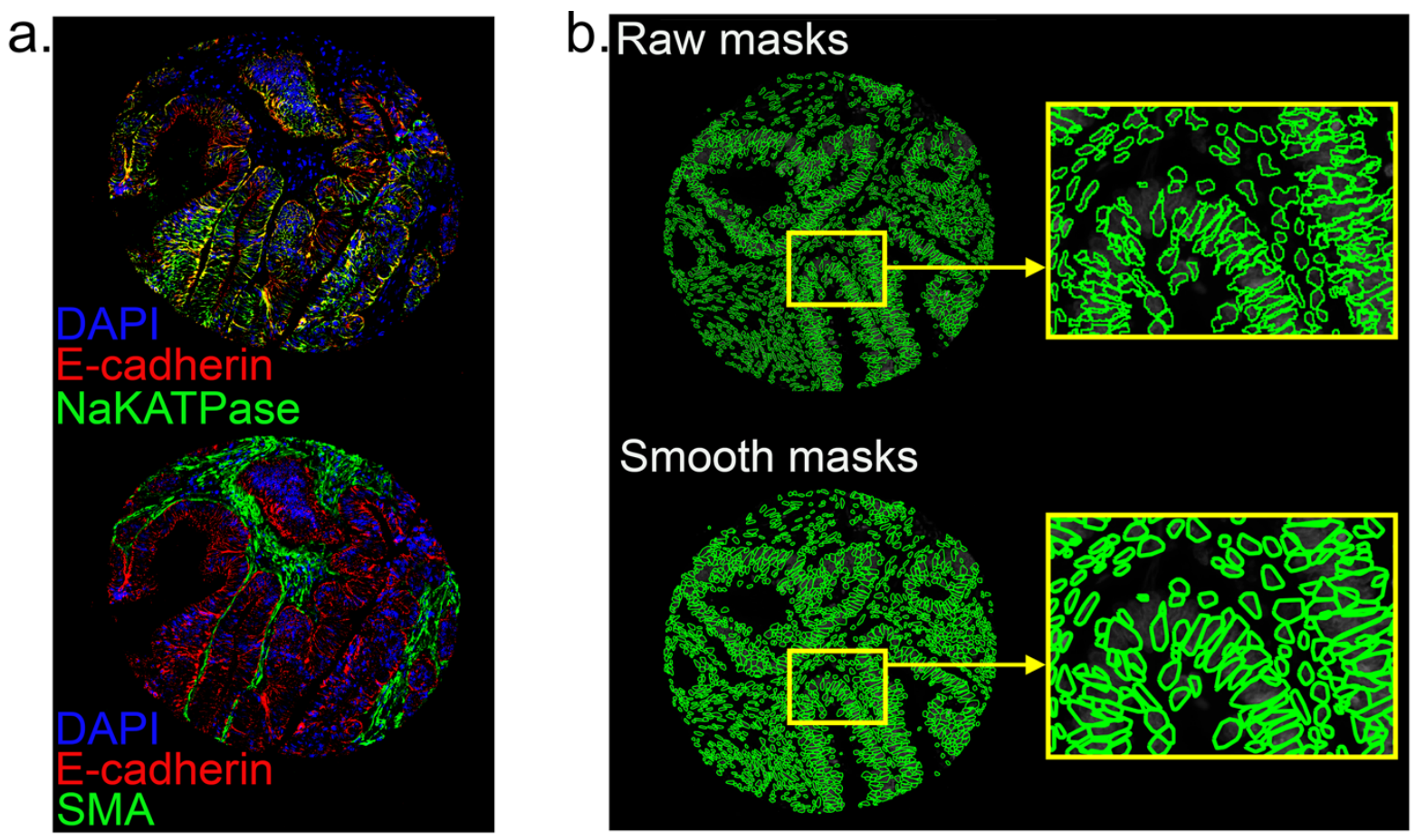

\section{Figure S2}

\begin{tabular}{|c|c|c|c|c|c|}
\hline Apoptosis & PI3K/AKT/MTOR & Adhesion and structure & Cell types and states & Oncogenic & PTM \\
\hline \multirow[t]{2}{*}{ c-CASP 3} & 4EBP1 & beta-actin & ALDH1 & beta-catenin & p4EBP1 \\
\hline & AKT1 & Claudin-1 & CD163 & c-MET & pEGFR \\
\hline Hedgehog & EGFR & Collagen IV & CD20 & $\operatorname{cox} 2$ & pERK \\
\hline \multirow[t]{2}{*}{$\mathrm{IHH}$} & EZH2 & E-cadherin & CD3 & ЕрСАМ & pGSK3a \\
\hline & FOXO3a & ЕрСАМ & CD31 & EZH2 & pGSK3b \\
\hline Hypoxia & p21 & Fibronectin & CD68 & & PI3Кp110a \\
\hline \multirow[t]{2}{*}{ CA9 } & p53 & $\operatorname{Lamin} \mathrm{A} / \mathrm{C}$ & CD79 & \begin{tabular}{|l|} 
Tumor suppressive \\
\end{tabular} & pMAPKAPK2 \\
\hline & PI3Kp110a & NaKATPase & EZH2 & F0X03a & PMET \\
\hline Mismatch repair & 56 & & Fibronectin & p53 & pS6 \\
\hline MLH1 & & Extracellular transport & NDRG1 & PTEN & p_p38MAPK \\
\hline MSH2 & RAS/RAF/MAPK & and metabolism & PCK26 & Wnt5a & \\
\hline p21 & EGFR & ALB & SMA & & \\
\hline \multirow[t]{2}{*}{ PCNA } & ERK1/2 & GLUT1 & & & \\
\hline & EZH2 & TKLT1 & & & \\
\hline
\end{tabular}

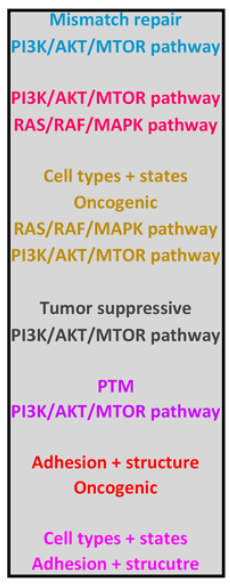




\section{Figure S3}

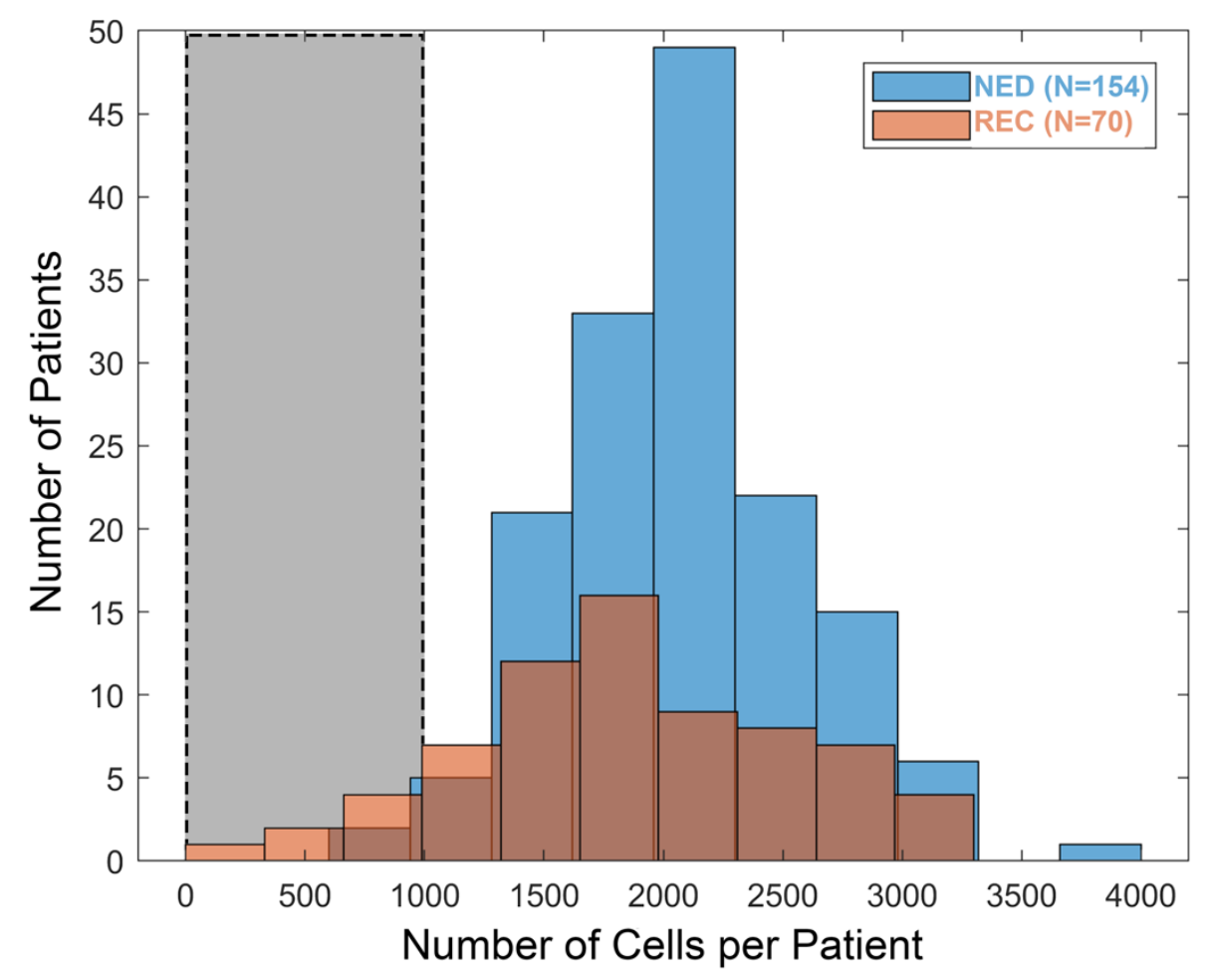

Figure S4

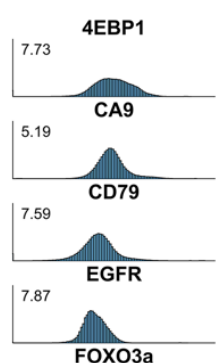

4.22

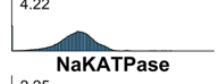

2.25

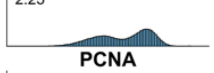

6.84

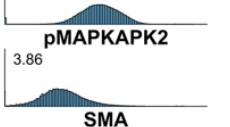

| 3.66
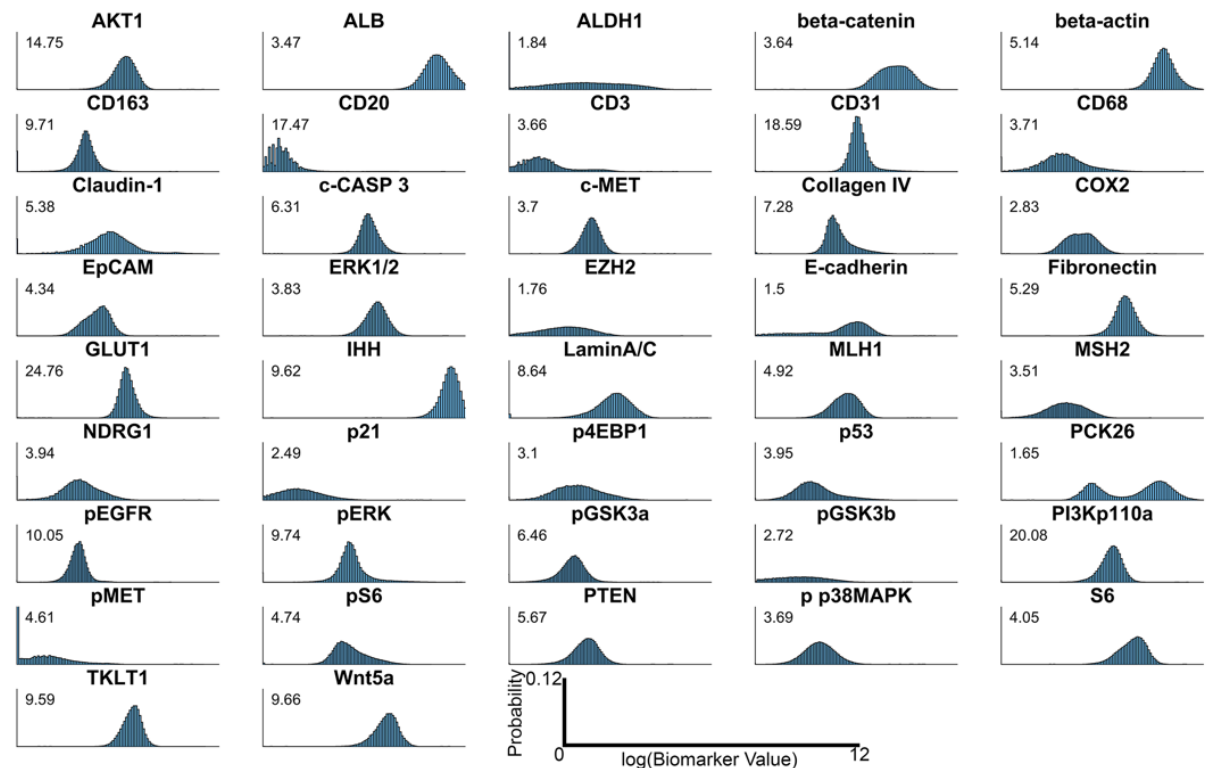
bioRxiv preprint doi: https://doi.org/10.1101/2020.10.02.322529; this version posted October 20, 2020. The copyright holder for this preprint (which was not certified by peer review) is the author/funder. All rights reserved. No reuse allowed without permission.

\section{Figure S5}

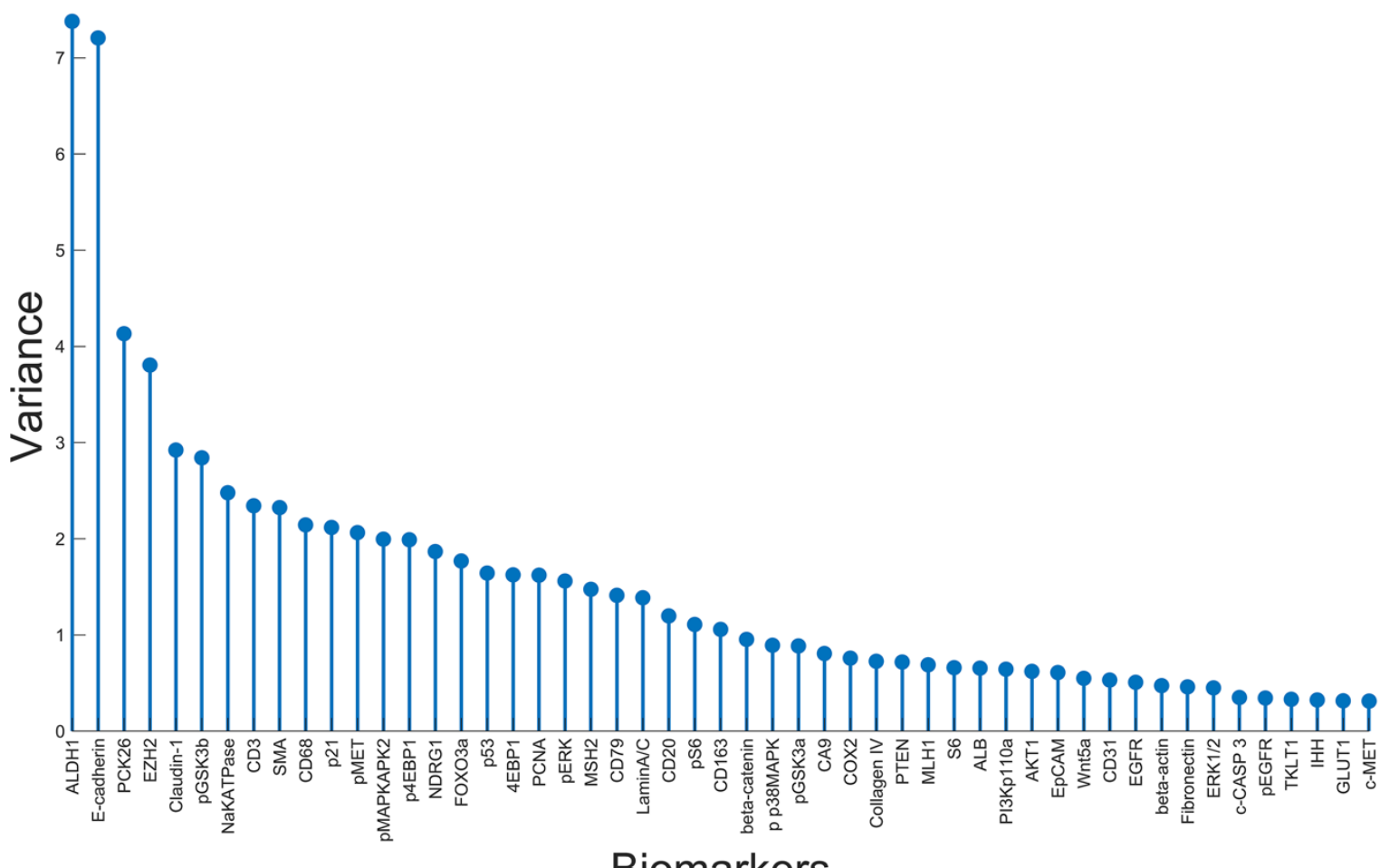

\section{Figure S6}

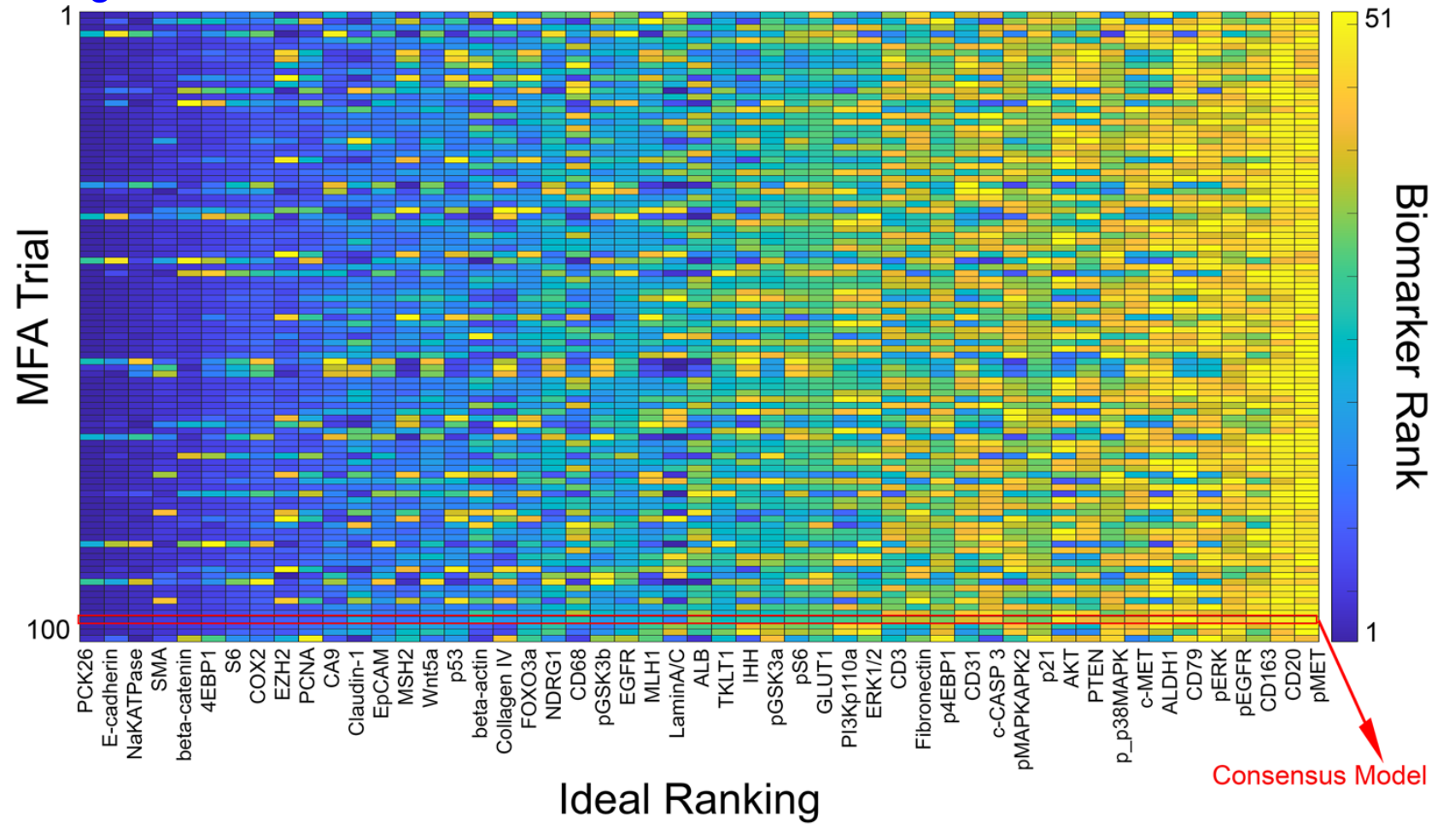


bioRxiv preprint doi: https://doi.org/10.1101/2020.10.02.322529; this version posted October 20, 2020. The copyright holder for this preprint (which was not certified by peer review) is the author/funder. All rights reserved. No reuse allowed without permission.

\section{Figure S7}
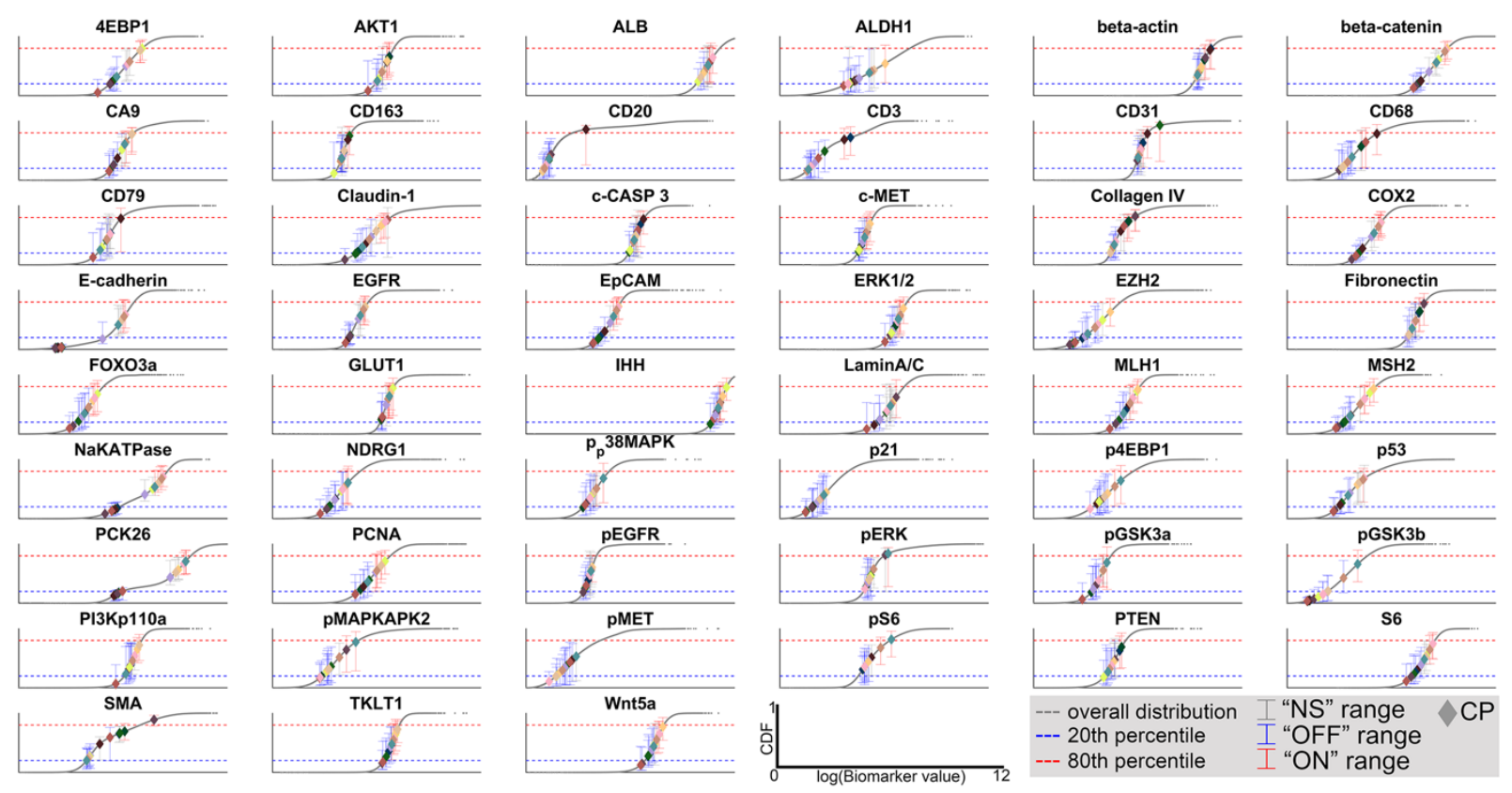


\section{Figure S8}
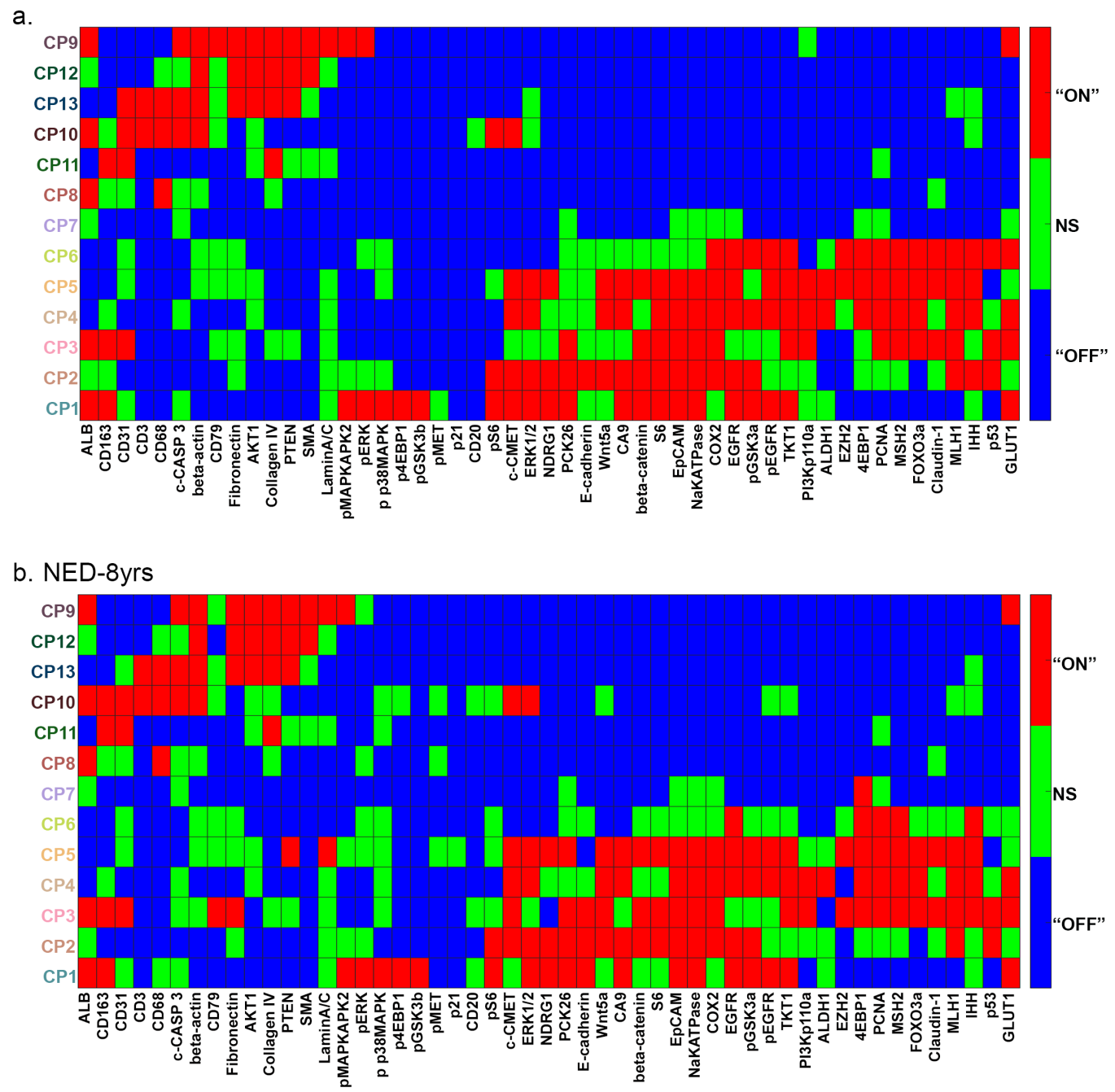

C. REC-3yrs

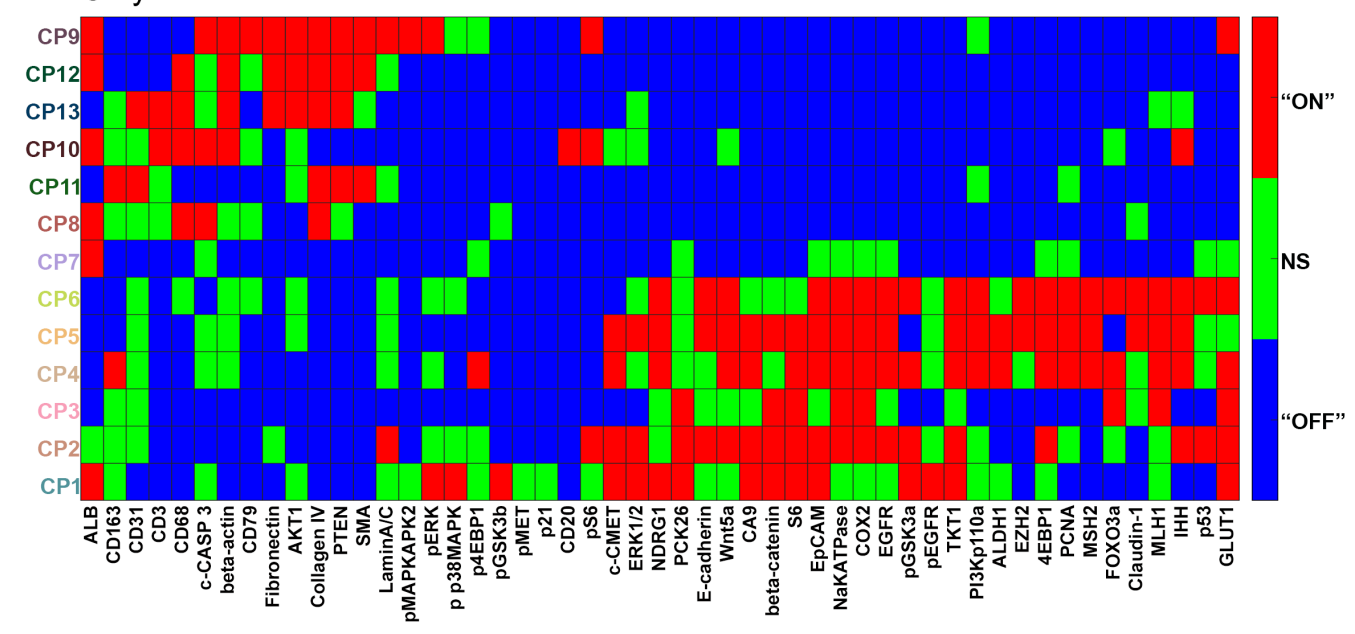




\section{Figure S9}

NED-8yrs REC-3yrs — Mean

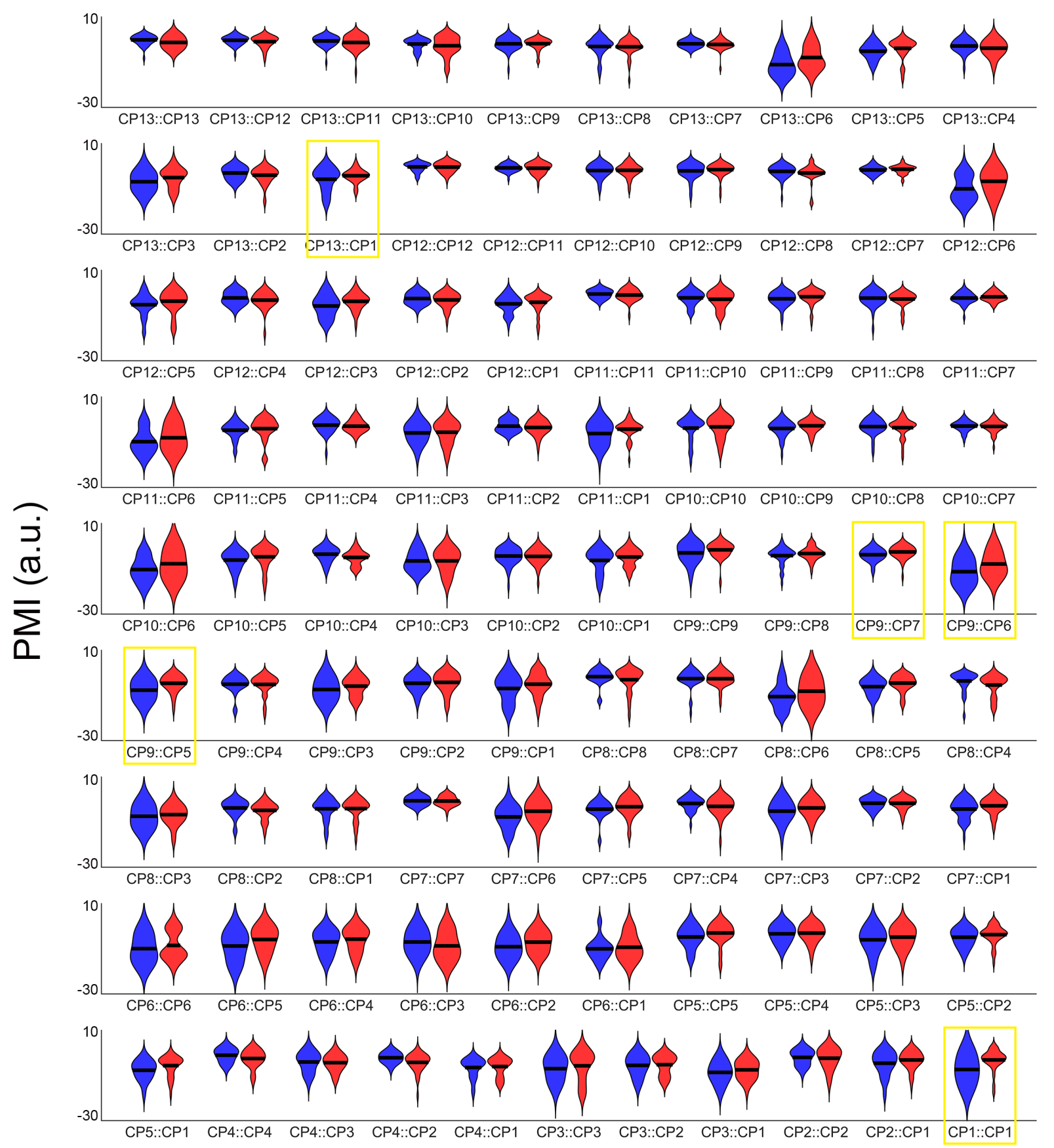




\section{Figure S10}

a.LEVEL 1: Epithelial-Stromal Dissection

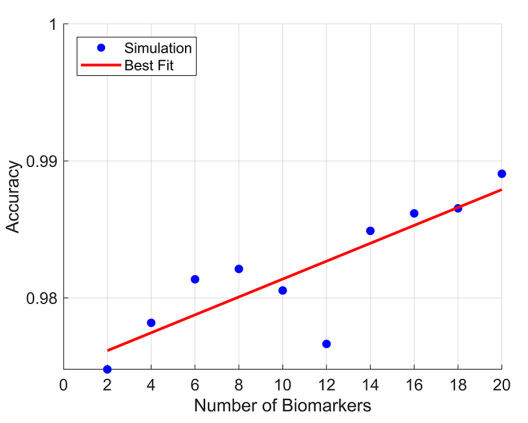

b.LEVEL 2: Stromal Sub-Cluster Dissection
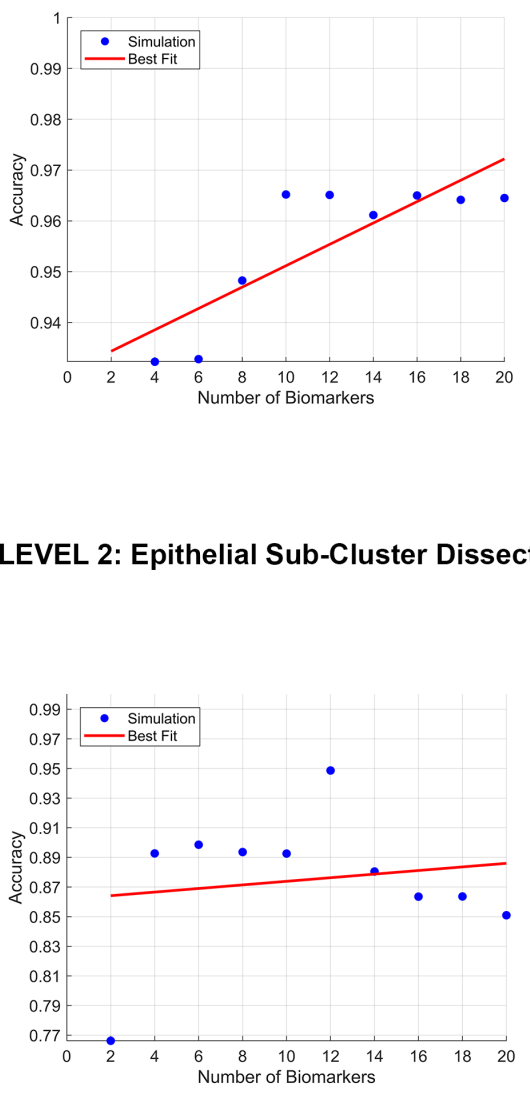
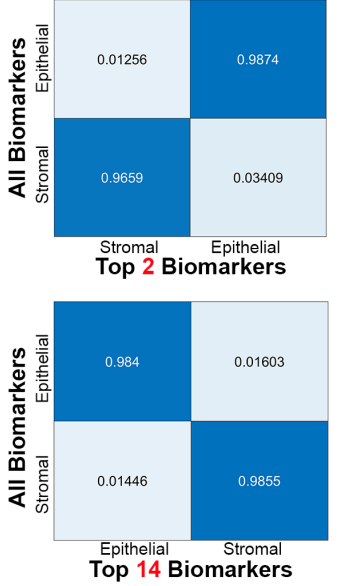

Top 14 Biomarkers

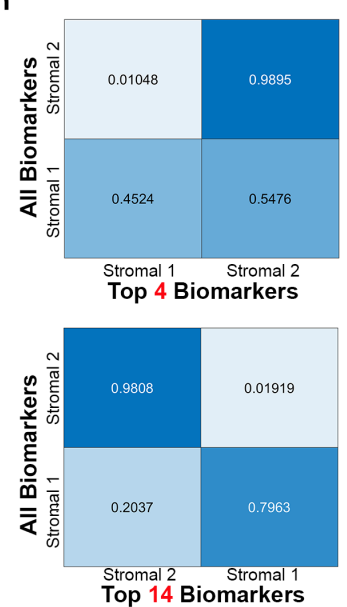

\section{c. LEVEL 2: Epithelial Sub-Cluster Dissection}
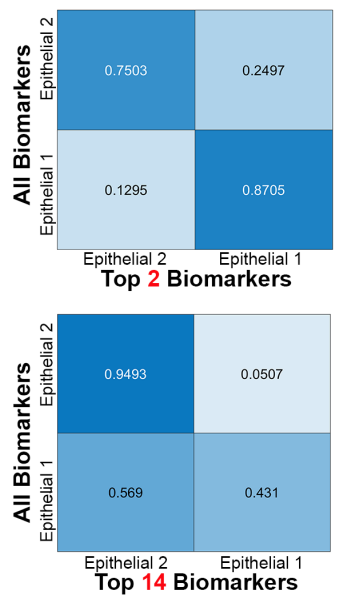
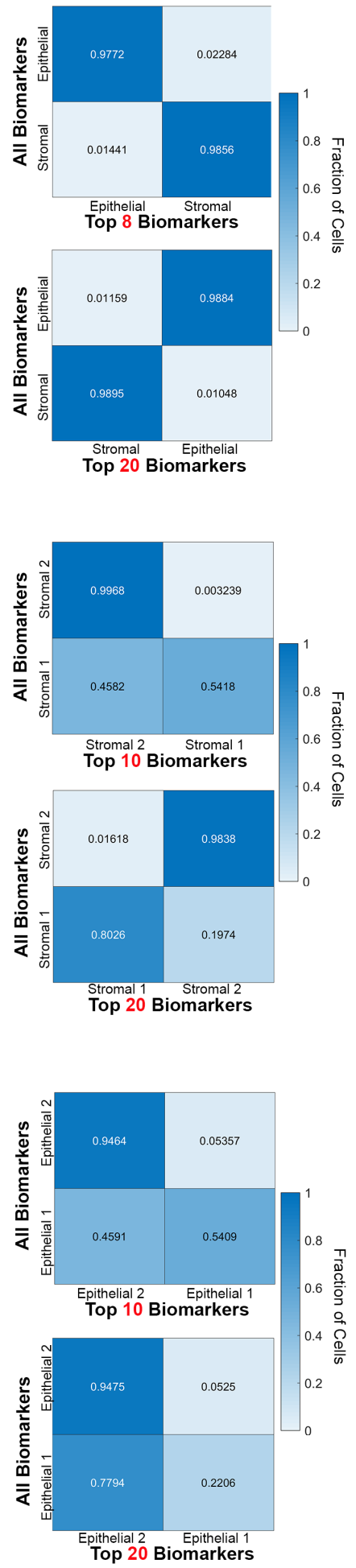